\title{
On quantum corrections in higher-spin theory in flat space
}

\section{Dmitry Ponomarev and Arkady A. Tseytlin ${ }^{1}$}

Theoretical Physics Group, Blackett Laboratory, Imperial College, London, SW7 2AZ, U.K.

E-mail: d.ponomarev@imperial.ac.uk, tseytlin@imperial.ac.uk

ABSTRACT: We consider an interacting theory of an infinite tower of massless higher-spin fields in flat space with cubic vertices and their coupling constants found previously by Metsaev. We compute the one-loop bubble diagram part of the self-energy of the spin 0 member of the tower by summing up all higher-spin loop contributions. We find that the result contains an exponentially UV divergent part and we discuss how it could be cancelled by a tadpole contribution depending on yet to be determined quartic interaction vertex. We also compute the tree-level four-scalar scattering amplitude due to all higherspin exchanges and discuss its inconsistency with the BCFW constructibility condition. We comment on possible relation to similar computations in AdS background in connection with AdS/CFT.

KEYwords: Higher Spin Gravity, AdS-CFT Correspondence

ARXIV EPRINT: 1603.06273

\footnotetext{
${ }^{1}$ Also at Lebedev Institute, Moscow.
} 


\section{Contents}

1 Introduction 1

$\begin{array}{lll}2 & \text { Free higher spin action } & 6\end{array}$

$\begin{array}{lll}3 & \text { Cubic interaction vertices } & 7\end{array}$

$\begin{array}{ll}3.1 \text { Deformation of the free action } & 7\end{array}$

3.2 Deformation of gauge transformations 9

$\begin{array}{lll}3.3 \text { Ghost action } & 10\end{array}$

$\begin{array}{llr}4 & \text { Feynman rules } & 11\end{array}$

5 Tree-level 4-scalar scattering amplitude $\quad 13$

$\begin{array}{ll}5.1 \text { Exchange contribution } & 14\end{array}$

$\begin{array}{ll}5.2 \text { Comments on 4-vertex contribution } & 15\end{array}$

$\begin{array}{lll}6 & \text { One-loop scalar self-energy correction } & \mathbf{1 7}\end{array}$

$\begin{array}{ll}6.1 & \text { Individual bubble diagrams } \\ 6.2 & 18\end{array}$

$\begin{array}{ll}6.2 \text { Summing over spins } & 19\end{array}$

$\begin{array}{ll}\text { 6.3 UV divergences and regularization } & 20\end{array}$

6.4 Comments on tadpole diagram contribution 22

$\begin{array}{lll}7 & \text { Concluding remarks } & 23\end{array}$

A Relation between covariant and light-cone cubic vertices $\quad 24$

A.1 Free fields in light-cone gauge 24

$\begin{array}{lll}\text { A.2 Cubic interactions } & 25\end{array}$

$\begin{array}{ll}\text { B Test of BCFW constructibility condition } & 27\end{array}$

$\begin{array}{ll}\text { C Details of summation over spins } & 28\end{array}$

\section{Introduction}

A possibility of an interacting theory of an infinite tower of massless higher spins in flat space is an old question with various no-go theorems prohibiting the existence of minimal (low-derivative) couplings or long-distance interactions (see [1] for a review). At the same 
time, non-trivial cubic vertices containing higher derivatives were constructed in the past using various approaches in $[2-16] .^{1}$

The allowed cubic vertices $\partial^{n} \phi_{s_{1}} \phi_{s_{2}} \phi_{s_{3}}$ have the number of derivatives $n$ constrained by $s_{2}+s_{3}-s_{1} \leq n \leq s_{2}+s_{3}+s_{1}$ (assuming $s_{1} \leq s_{2} \leq s_{3}$ ). Remarkably, the coupling constants in these vertices can be fixed in terms of a single dimensional constant $\ell$ [6]. For example, for the highest-derivative vertex one gets $g_{s_{1} s_{2} s_{3}} \sim \frac{\ell^{s_{1}+s_{2}+s_{3}-1}}{\left(s_{1}+s_{2}+s_{3}-1\right) !}$. Assuming that a Noether deformation procedure can completely determine also the quartic and higher vertices one may conjecture the existence of a theory containing an infinite tower of massless Fronsdal fields $\phi_{s}(s=0,1,2, \ldots, \infty)$ with an action depending on one dimensionless coupling $g$ and one dimensional parameter $\ell$ and having the following structure ${ }^{2}$

$$
\begin{aligned}
S_{\text {flat }} & =\frac{1}{g^{2}} \int d^{4} x\left[\sum_{s} \phi_{s} \partial^{2} \phi_{s}+\sum \ell^{n-1} \partial^{n} \phi_{s_{1}} \phi_{s_{2}} \phi_{s_{3}}+\sum \ell^{k-2} \partial^{k} \phi^{4}+\ldots\right] \\
& \rightarrow \int d^{4} x\left[\sum_{s} \phi_{s} \partial^{2} \phi_{s}+g \sum \ell^{n-1} \partial^{n} \phi_{s_{1}} \phi_{s_{2}} \phi_{s_{3}}+g^{2} \sum \ell^{k-2} \partial^{k} \phi^{4}+\ldots\right] .
\end{aligned}
$$

Here $g$ controls the expansion in number of fields (and also loop expansion) while $\ell$ sets up an effective scale (i.e. it appears together with derivatives or momenta). Such a theory is effectively non-local as the number of derivatives is unbounded given that spins can take any value up to infinity.

One may wonder why such an unusual theory (assuming it indeed exists) may be of any interest. One reason is that it may have hidden simplicity due to its large gauge (and global) higher spin symmetry. For example, the free theory $\sum_{s=0}^{\infty} \int d^{4} x \phi_{s} \partial^{2} \phi_{s}$ turns out to have zero total number of effective dynamical degrees of freedom and thus a trivial partition function $Z=1$ [24]. ${ }^{3}$ This is true if one uses a particular prescription of summation

\footnotetext{
${ }^{1}$ How some constraints of no-go theorems may be avoided is discussed in $[1,17,18]$. The ColemanMandula theorem that prohibits existence of higher spin conserved charges assumes finite number of particles of mass below certain scale and analyticity of the amplitudes. Weinberg's soft theorem [19] is a requirement of linearized gauge invariance with respect to the spin $s$ leg in the amplitude. It is satisfied automatically if 3-point vertices are gauge-invariant on-shell as is the case for the known flat space higher spin vertices discussed below. In general, the soft theorem constraines a theory with $s-s^{\prime}-s^{\prime}$ couplings with minimal number $s$ of derivatives in the vertex. It imposes conditions on scattering amplitudes with at least one higher spin $s$ particle on an external line which are equivalent to conservation of higher spin charges (which are higher than quadratic in external momentum and thus allow only elastic scattering with permutations of momenta). It leads to non-trivial constraints only if exchanged spins are lower than external ones [17]. Constraints on cubic couplings based on the assumption of BCFW constructibility [20] applied to massless 4-point scattering amplitude [21-23] may not apply if one allows for some non-locality of the 4-point vertex in a theory that contains infinite sum over spins/derivatives. It is likely that the condition of BCFW constructibility is stronger than that of the existence of an interacting theory constructed from a free theory via gauge symmetry deformation or Noether method (see also discussion below).

${ }^{2}$ Here we will consider the case of $4 \mathrm{~d}$ space-time (so that $\phi_{s}$ are assumed to have dimension length ${ }^{-1}$ ) but most of the discussion below will be true also in any dimension. In $d=4$ the light-cone gauge vertices of [6] that admit a local covariant generalization contain only two structures: with maximal $s_{2}+s_{3}+s_{1}$ and with minimal $s_{2}+s_{3}-s_{1}$ numbers of derivatives.

${ }^{3}$ In contrast to supersymmetric theories here $Z=1$ even at finite temperature (all bosonic fields have the same statistics) so closer analogy is with a topological field theory. A non-trivial generalization of $Z$ to quotients of flat space in the presence of angular potentials was discussed in [25].
} 
over spins that should be consistent with underlying symmetry of the theory. One may then expect that under such summation prescription other quantum corrections may also be simple. ${ }^{4}$ For example, despite having a dimensional coupling $\ell$ the theory (1.1) may actually be UV finite. This would be analogous to what happens in string theory viewed as a collection of a few massless and an infinite set of massive higher spin fields where a particular prescription of summation over all contributions implied by the underlying world-sheet formulation leads to the UV finiteness of scattering amplitudes. ${ }^{5}$

Another motivation to study (1.1) is its possible relation to the massless higher-spin theory in AdS space. The existence of the consistent cubic couplings (containing also lowderivative or minimal-coupling parts and avoiding the no-go theorems) was first pointed out in $[29,30]$. One may conjecture that elimination of an infinite set of auxiliary fields present in the non-linear Vasiliev's equations [31,32] expanded near the AdS vacuum may lead to an action for the tower of physical massless Fronsdal fields that has a structure similar to that of (1.1) (cf. [33-35])

$$
S_{\mathrm{AdS}}=\frac{1}{g^{2}} \int d^{4} x\left[\sum_{s} \phi_{s}\left(\nabla^{2}+\ldots\right) \phi_{s}+\sum L^{n-1} \nabla^{n} \phi_{s_{1}} \phi_{s_{2}} \phi_{s_{3}}+\sum L^{k-2} \nabla^{k} \phi^{4}+\ldots\right] .
$$

Here $L$ is the AdS radius and the cubic vertices now contain also the low-derivative ("minimal-coupling") tail of terms (e.g. $n=1,2, \ldots$ ). To understand a possible reason for their presence let us imagine that the action (1.1) could admit a generalization to a curved background where the flat metric would be replaced by a curved one $g_{a b}$ and the flat derivatives $\partial_{a}$ by the covariant ones $\nabla_{a}$. Then in addition to the cubic terms $\ell^{n-1} \nabla^{n} \phi_{s_{1}} \phi_{s_{2}} \phi_{s_{3}}$ as in (1.1) with $n \geq s_{2}+s_{3}-s_{1}$ one could also have terms of the same dimension with less derivatives but with extra powers of the curvature, $\ell^{n-1} R^{k} \nabla^{n-2 k} \phi_{s_{1}} \phi_{s_{2}} \phi_{s_{3}}$, i.e. $\ell^{n-1} L^{-2 k} \nabla^{n-2 k} \phi_{s_{1}} \phi_{s_{2}} \phi_{s_{3}}$ in the case of the AdS space with $R \sim L^{-2}$. In the action (1.2) corresponding to the Vasiliev-type theory there is just one dimensional scale, i.e. $\ell$ is effectively identified with the curvature scale $L$ and thus the flat space limit $(L \rightarrow \infty)$ is formally singular. ${ }^{6}$

While a naive flat-space limit that leads from (1.2) to (1.1) may not exist, there may be still some formal procedure of relating the two actions in which these low-derivative couplings would decouple and with $L$ in (1.2) being effectively replaced by another scale $\ell$ in (1.1). One might think that as the leading short-distance behaviour should be controlled

\footnotetext{
${ }^{4}$ Similar simplifications were observed in conformal higher-spin theory [24, 26, 27]. The existence of higher spin conserved charges may also have drastic consequences for the S-matrix with higher spin particles on external lines (as in the case of integrable theories in 2 dimensions [28]).

${ }^{5}$ At the same time, it seems unlikely that (1.1) may be consistently related to a zero-tension limit of the bosonic string theory combined with a truncation to the leading Regge trajectory: zero-tension limit does not appear to be well-defined in flat space and, moreover, the tower of fields on the leading Regge trajectory may not lead to a consistent UV theory on its own.

${ }^{6}$ Here we assumed that the curved metric (e.g., $d s_{\text {AdS }}^{2}=d u^{2}+e^{-u / L} d x^{m} d x_{m}$ ) has a smooth flat space limit without need to rescale the coordinates. In general, one may contemplate taking a flat space limit that involves some singular rescalings of the fields, coordinates and coupling constants (e.g., as in [12, 36]). It is not clear if such a limit may actually exist beyond the cubic interaction level given that quartic vertices may contain sums over all orders in derivatives for fixed external spins.
} 
by the highest derivative terms, one may then expect that the UV properties of a flat-space and a curved-space (e.g. AdS) theories may be similar. This, however, may not be true in the present context of an effectively non-local theory (containing all powers of derivatives in the vertices due to summation over an infinite set of higher spin fields). Still, the study of a simpler theory (1.1) may shed some light on some properties of (1.2).

The definition of a quantum theory with an infinite set of fundamental fields like the one in (1.1) or (1.2) is a priori ambiguous as the sum of individual field contributions over all spins may be divergent. This ambiguity is to be fixed in a way that is consistent with preservation of underlying higher spin symmetry as was recently discussed in $[26,27,37$ 42]. In particular, under a special summation prescription the free-field partition function of the AdS theory (1.2) is trivial, i.e. $Z=1[38,39]$, just as in the flat space theory (1.1) [24].

This definition should be consistent with the vectorial AdS/CFT duality [43-45] between the massless higher spin theory in $\mathrm{AdS}_{d+1}$ and singlet sector of the free $\mathrm{U}(N)$ or $O(N)$ scalar CFT in $\mathbb{R}^{d}$ which is also controlled by the underlying higher spin symmetry. This duality was tested at the tree level for some 3-point functions [33, 46] which are essentially fixed by unbroken higher spin symmetry [47]. ${ }^{7}$ Let us note that some cubic vertices for the Fronsdal fields derived from the Vasiliev's theory upon elimination of auxiliary fields are formally divergent $[33,35,46]$ and thus require additional definition/regularization even before summation over all physical spins.

Alternatively, one can try to reconstruct the cubic and quartic action (1.2) (with $\left.g^{2}=1 / N\right)$ by requiring that it should reproduce the boundary CFT correlators at the tree level [51-53] (for related earlier work see [54]). Then a crucial test of the duality will be to check that all quantum corrections to the "on-shell" value of the effective action of the theory (1.2) should vanish since the correlators of conserved currents in the free boundary CFT do not receive $1 / N$ corrections, i.e. are given exactly by their large $N$ values. $^{8}$ Assuming that the matching of correlators at separated points may be extended also to integrated correlators, this suggests, in particular, that the vacuum partition function of the AdS theory (1.2) should vanish not just at the leading one-loop order [38] but also to all orders in $g=1 / \sqrt{N}$ expansion.

Another non-trivial quantum test would be the demonstration of the vanishing of the 1-loop correction to the spin $s$ field 2-point function - the 2-point functions or dimensions of the currents in the boundary CFT should not be $1 / N$-corrected in the case of unbroken higher spin symmetry. This "self-energy" correction is given by the sum of the two types of one-loop Witten diagrams: the "bubble" diagram (with two bulk-to-bulk propagators and two cubic vertices) and the "tadpole" diagram determined by the quartic vertex in (1.2). ${ }^{9}$

\footnotetext{
${ }^{7}$ Also, all $n$-point functions of the free CFT have been identified with suitable invariants in the Vasiliev theory in $[48-50]$.

${ }^{8} \mathrm{~A}$ priori it is possible that quantum corrections to the effective action of the theory (1.2) could be non-vanishing but having such special "local" form that they do not contribute to derivatives over the boundary sources taken at separated points. At the same time, this seems unlikely as quantum corrections should be controlled by the higher spin symmetry that should constrain also possible contact terms.

${ }^{9}$ Some one-loop corrections to propagators in AdS were computed previously for spin 2 in $[55,56]$ and for higher spins in [57]. One-loop computations based on Mellin representation were performed in [58]. Related discussions from higher spin AdS/CFT perspective appeared in [59-62].
} 
With this motivation in mind here we will address a simpler question about the one-loop bubble-diagram part of the self-energy correction in the higher spin flat space theory (1.1). Certain features of the flat-space result should be similar to the ones in the AdS case, at least in what concerns the leading UV behaviour. Our aim will be to extract the UV divergence of the bubble diagram and to see if it can be cancelled against a tadpole contribution coming from (yet unknown) 4-point vertex.

For simplicity, we shall consider the case when only the scalar particle (spin 0 member of the higher spin tower) appears on the two external lines of the self-energy diagram. In this case the bubble graph contribution is determined by the 3 -point vertex $0-s_{2}-s_{3}$ (containing $n=s_{2}+s_{3}$ derivatives) which is essentially unique. We shall use the explicit value of its coefficient $\sim \frac{\ell^{s_{2}+s_{3}-1}}{\left(s_{2}+s_{3}-1\right) !}$ found in [6] and perform the summation over all spins in the loop. ${ }^{10}$ The one-loop diagram will then be quadratic in dimensionless coupling $g$ in (1.1) and given by the virtual momentum integral with non-trivial dependence on the product of $\ell$ with external momentum.

To find the full result for the $g^{2}$ correction to self-energy diagram one is to add also the ghost-loop contribution and the tadpole graph contribution. The latter requires the knowledge of the 0-0-s-s quartic vertex (and similar one for the ghosts). The problem of determining quartic interactions of massless higher spins in flat and AdS spaces was addressed using different approaches in, e.g., [6, 14, 17, 52, 67-70], but its full conclusive solution is yet to be found. While being unable to determine the tadpole contribution explicitly here we shall still comment on its expected UV behaviour required to cancel the UV divergent part of the bubble graph. We shall also note that the tadpole contribution is not expected to alter the non-trivial (non-analytic) external momentum dependence coming from the bubble graph.

The rest of this paper is organized as follows. In section 2 we shall define the free Fronsdal action for totally symmetric massless higher spin fields in flat $d$ dimensions and impose the de Donder gauge. In section 3 we shall discuss the structure of the $0-s_{2}-s_{3}$ cubic vertices required for subsequent computations. We shall also consider the leading term in the deformation of the gauge transformations due to the presence of the cubic interactions and thus determine the corresponding quadratic and cubic terms in the ghost action. In section 4 we shall review the derivation of the higher spin propagator in the de Donder gauge and describe the resulting Feynman rules.

Section 5 will be devoted to the computation of the tree-level 4-scalar scattering amplitude. We shall first explicitly compute the exchange part of the amplitude and then comment on possible contribution of the 4-scalar "contact" vertex. In section 6 we shall compute the bubble diagram contribution to the one-loop scalar self-energy corrections and discuss its UV behaviour. We shall then discuss a possibility that UV divergence of the

\footnotetext{
${ }^{10}$ This cubic vertex can be found, e.g., by requiring the gauge invariance of the full non-linear action to the lowest order in the coupling $g$. At this order the coupling constants of individual vertices for different spins are independent. By requiring gauge invariance to the next $g^{2}$ order, these coupling constants can all be expressed in terms of a unique dimensionful parameter $\ell$ as in (1.1). An equivalent analysis was carried out in flat $4 \mathrm{~d}$ space in the light-cone approach in [6]. In AdS similar relations were found within the FradkinVasiliev approach $[30,63,64]$ by imposing the Jacobi identity for the gauge symmetry deformations $[65,66]$.
} 
bubble diagram may be cancelled against a tadpole graph contribution determined by the 4-point vertex. Some concluding remarks will be made in section 7 .

In appendix $\mathrm{A}$ we shall explain the relation between the covariant cubic vertex we use and the light-cone gauge cubic vertex in [6]. In appendix B we shall discuss whether these vertices are consistent with the BCFW constructibility condition (see also comments at the end of section 5). Appendix $\mathrm{C}$ will contain some details of the computation of sums over spins in section 6 .

\section{$2 \quad$ Free higher spin action}

To make expressions more compact we shall represent the totally symmetric higher spin tensor fields by

$$
\phi_{s}(x, u)=\frac{1}{s !} \phi_{s}^{a_{1} \ldots a_{s}}(x) u_{a_{1}} \ldots u_{a_{s}},
$$

where $u_{a}$ is an arbitrary constant vector. Then the Fronsdal action [71] may be written as ${ }^{11}$

$$
S^{(2)}\left[\phi_{s}\right]=\frac{s !}{2} \int d^{d} x\left[\phi_{s}\left(x, \partial_{u}\right) \hat{T} \hat{\mathcal{F}} \phi_{s}(x, u)\right]_{u=0},
$$

where

$$
\hat{T} \equiv 1-\frac{1}{4} u^{2} \partial_{u}^{2}, \quad \hat{\mathcal{F}} \equiv \partial_{x}^{2}-\left(u \cdot \partial_{x}\right) \hat{D}, \quad \hat{D} \equiv\left(\partial_{x} \cdot \partial_{u}\right)-\frac{1}{2}\left(u \cdot \partial_{x}\right) \partial_{u}^{2},
$$

and the off-shell field $\phi_{s}$ is assumed to be double-traceless, i.e. satisfying $\left(\partial_{u}^{2} \equiv \eta^{a b} \frac{\partial}{\partial u^{a}} \frac{\partial}{\partial u^{b}}\right)$

$$
\left(\partial_{u}^{2}\right)^{2} \phi_{s}(x, u)=0 .
$$

As in the second line of (1.1) we assume that the dimensionless coupling $g$ is absorbed into $\phi_{s}$ so that it will appear in the interaction vertices.

The equation of motion for $(2.2)$ is

$$
\frac{\delta S^{(2)}\left[\phi_{s}\right]}{\delta \phi_{s}}=\hat{T} \hat{\mathcal{F}} \phi_{s}(x, u) \approx 0
$$

Here and in what follows we use the symbol $\approx$ to denote the equalities that hold modulo terms proportional to the free equations of motion. By noting that $\hat{T}$ is invertible

$$
\hat{T}^{-1} \equiv 1-\frac{1}{2 d+4 u \cdot \partial_{u}-12} u^{2} \partial_{u}^{2}, \quad \hat{T}^{-1} \hat{T} \phi_{s}(x, u)=\hat{T} \hat{T}^{-1} \phi_{s}(x, u)=\phi_{s}(x, u),
$$

one finds that the free equations (2.5) can be equivalently rewritten as

$$
\hat{\mathcal{F}} \phi(x, u) \approx 0 .
$$

Note, that in (2.6) we kept only those terms in $\hat{T}^{-1}$ that do not annihilate double-traceless tensors, i.e. do not contain $\left(\partial_{u}^{2}\right)^{n}$ with $n>1$ (cf. (2.4)).

\footnotetext{
${ }^{11}$ The action (2.2) is canonically normalised and its overall sign is chosen appropriately to ensure positive energy of field fluctuations for the mostly plus Minkowski metric $\eta=\operatorname{diag}(-,+,+, \ldots,+)$.
} 
The Fronsdal action (2.2) is invariant under the gauge transformations

$$
\delta_{s}^{(0)} \phi_{s}(x, u)=\left(u \cdot \partial_{x}\right) \varepsilon_{s-1}(x, u),
$$

with the traceless gauge parameter $\varepsilon_{s-1}(x, u)$

$$
\varepsilon_{s-1}(x, u) \equiv \frac{1}{(s-1) !} \varepsilon_{s-1}^{a_{1} \ldots a_{s-1}}(x) u_{a_{1}} \ldots u_{a_{s-1}}, \quad \partial_{u}^{2} \varepsilon_{s-1}(x, u)=0 .
$$

It is convenient to impose the de Donder gauge

$$
\hat{D} \phi_{s}(x, u)=0 \text {. }
$$

In this gauge the Fronsdal operator $\hat{\mathcal{F}}$ in $(2.3)$ simplifies so that the action $(2.2)$ becomes

$$
S^{(2)}\left[\phi_{s}\right]=\frac{s !}{2} \int d^{d} x\left[\phi_{s}\left(x, \partial_{u}\right) \hat{T} \partial_{x}^{2} \phi_{s}(x, u)\right]_{u=0},
$$

while the equations of motion (2.7) take the form

$$
\square \phi_{s}(x, u) \approx 0, \quad \square=\partial_{x}^{2} .
$$

\section{Cubic interaction vertices}

In this section we shall present cubic vertices for the physical fields $\phi_{s}$ and the ghosts corresponding to the de Donder gauge (2.10). To construct cubic vertices in the covariant form one usually starts by specifying their traceless transverse parts. Then these vertices can be completed to full-fledged off-shell ones [13-16]. For our purposes it is not necessary to face the difficulties inherent to general off-shell interactions as it suffices to know the vertices in the de Donder gauge. Moreover, as here we will be interested in computing diagrams with only spin 0 particles on external lines we may restrict consideration to cubic vertices with one of the fields having $s=0$. In this case it turns out that the tracelesstransverse vertices give already the consistent vertices in the de Donder gauge, i.e. do not require any completion. ${ }^{12}$

\subsection{Deformation of the free action}

Adding cubic interaction part $S^{(3)}$ to the free action $S^{(2)}$ and requiring gauge invariance of the combined non-linear action gives at the first non-trivial order the condition

$$
\delta^{(0)} S^{(3)}+\delta^{(1)} S^{(2)}=0,
$$

where $\delta^{(1)}$ is a deformation of the gauge transformation (2.8) which is linear in the fields $\phi_{s}$. The first term in (3.1) thus vanishes modulo the free equations of motion (2.5), i.e.

$$
\delta^{(0)} S^{(3)} \approx 0 .
$$

\footnotetext{
${ }^{12}$ The observation that the traceless-transverse vertices are sufficient to find the consistent vertices in the de Donder gauge was already made in [13-16]. In the most general case in the de Donder gauge apart from traceless-transverse contribution there is also a term which is cubic in traces [13]. In our case it is absent and this is indeed an extra simplification that we use.
} 
Once the cubic vertex is found, the associated deformation of the gauge transformation can be extracted from (3.1).

The traceless-transverse part of the cubic vertex involving the spin 0 field and any two other higher spin fields can be written as $[13,14,16,36]$

$$
\begin{aligned}
S^{(3)}\left[\phi_{0}, \phi_{s_{2}}, \phi_{s_{3}}\right]=g_{0 s_{2} s_{3}} \int d^{d} x[ & \left(\partial_{u_{2}} \cdot \partial_{x_{31}}\right)^{s_{2}}\left(\partial_{u_{3}} \cdot \partial_{x_{12}}\right)^{s_{3}} \\
& \left.\times \phi_{0}\left(x_{1}\right) \phi_{s_{2}}\left(x_{2}, u_{2}\right) \phi_{s_{3}}\left(x_{3}, u_{3}\right)\right]_{\substack{u_{i}=0 \\
x_{i}=x}},
\end{aligned}
$$

where $\partial_{x_{i j}} \equiv \partial_{x_{i}}-\partial_{x_{j}}$ and $g_{0 s_{2} s_{3}}$ is a coupling constant. To show that it gives a consistent vertex in the de Donder gauge, let us verify that it satisfies (3.2). Using that

$$
\left[\partial_{u_{2}} \cdot \partial_{x_{31}}, u_{2} \cdot \partial_{x_{2}}\right]=\left(\partial_{x_{3}}-\partial_{x_{1}}\right) \cdot \partial_{x_{2}}=-\partial_{x_{3}}^{2}+\partial_{x_{1}}^{2}+\text { t.d. }
$$

where "t.d." stands for a total derivative term, we find

$$
\begin{aligned}
& \delta_{s_{2}}^{(0)} \phi_{s_{2}} \frac{\delta S^{(3)}\left[\phi_{0}, \phi_{s_{2}}, \phi_{s_{3}}\right]}{\delta \phi_{s_{2}}}=g_{0 s_{2} s_{3}} s_{2} \int d^{d} x[\left(\partial_{u_{2}} \cdot \partial_{x_{31}}\right)^{s_{2}-1}\left(\partial_{u_{3}} \cdot \partial_{x_{12}}\right)^{s_{3}}\left(\partial_{x_{1}}^{2}-\partial_{x_{3}}^{2}\right) \\
&\left.\times \phi_{0}\left(x_{1}\right) \varepsilon_{s_{2}-1}\left(x_{2}, u_{2}\right) \phi_{s_{3}}\left(x_{3}, u_{3}\right)\right]_{\substack{u_{i}=0 \\
x_{i}=x}} \approx 0 .
\end{aligned}
$$

This leading-order deformation analysis fixes the structure of the cubic vertices but leaves the coupling constants $g_{0 s_{2} s_{3}}$ in (3.3) undetermined. This happens because the gauge invariance conditions (3.1), (3.2) are linear in the deformation $S^{(3)}$. To find $g_{0 s_{2} s_{3}}$ one needs to consider higher-order deformations and to solve analogous higher-order constraints which become non-linear in the fields. At the next order one gets the condition

$$
\delta^{(0)} S^{(4)}+\delta^{(1)} S^{(3)}+\delta^{(2)} S^{(2)}=0,
$$

which involves quartic vertices $S^{(4)}$.

A conclusive analysis of higher-spin interactions in the covariant form at this quartic order was not performed so far, but to fix $g_{0 s_{2} s_{3}}$ we may use the result of Metsaev [6] obtained in $d=4$ in the light-cone gauge approach. Making the most general ansatz for the cubic interaction vertex and requiring the closure of the Poincare algebra to the $g^{2}$ order it was found [6] that all cubic couplings can be expressed in terms of a single parameter. In appendix A we will establish a dictionary between the light-cone cubic vertices and the covariant ones in the de Donder gauge and show that the result of $[6,7]$ when translated into the covariant language implies that

$$
g_{0 s_{2} s_{3}}=g \frac{\ell^{s_{2}+s_{3}-1}}{\left(s_{2}+s_{3}-1\right) !} .
$$

Here $g$ is an overall dimensionless coupling counting the power of fields and $\ell$ is a unique dimensional parameter (cf. (1.1)). Here $g_{000}=0$, i.e. there is no cubic scalar self-coupling.

Remarkably, the same expression for the cubic couplings (3.7) appears also in the action (1.2) for the massless higher spin fields in $\mathrm{AdS}_{4}$ which was reconstructed from the 
condition of consistency with the vectorial AdS/CFT duality. Namely, in [59] it was noted that $g_{00 s}$ couplings (3.7) agree with their AdS counterparts reconstructed from the free boundary CFT [52] and it was further conjectured that the agreement should hold also for general trilinear couplings of higher-spin gauge fields. This was indeed confirmed recently in [53]. This gives hope that some relation between the AdS action (1.2) and the flat space one (1.1) may hold even beyond the cubic order (despite a naive flat-space limit of (1.2) being singular). ${ }^{13}$

\subsection{Deformation of gauge transformations}

In addition to cubic interactions of the physical fields we need also to find similar vertices involving the ghost fields as these are required to compute the one-loop self-energy graphs. To find the ghost action corresponding to the de Donder gauge we need to know the deformation of the free gauge transformations (2.8) induced by the presence of the cubic vertex (3.3). ${ }^{14}$

This deformation may be found using (3.1). In order to compensate for the term containing $\partial_{x_{3}}^{2}$ in (3.5) one has to deform the spin $s_{3}$ field gauge transformation. In general, one has

$$
\delta_{s_{2}}^{(1)} \phi_{s_{3}} \frac{\delta S^{(2)}\left[\phi_{s_{3}}\right]}{\delta \phi_{s_{3}}}=s_{3} ! \int d^{d} x\left[\delta_{s_{2}}^{(1)} \phi_{s_{3}}\left(x_{3}, \partial_{u_{3}}\right) \hat{T}_{3} \partial_{x_{3}}^{2} \phi_{s_{3}}\left(x_{3}, u_{3}\right)\right]_{u_{3}=0} .
$$

Substituting (3.5) and (3.8) into (3.1) we find

$$
\begin{aligned}
\int d^{d} x[ & -g_{0 s_{2} s_{3}} s_{2}\left(\partial_{u_{2}} \cdot \partial_{x_{31}}\right)^{s_{2}-1}\left(\partial_{u_{3}} \cdot \partial_{x_{12}}\right)^{s_{3}} \phi_{0}\left(x_{1}\right) \varepsilon_{s_{2}-1}\left(x_{2}, u_{2}\right) \partial_{x_{3}}^{2} \phi_{s_{3}}\left(x_{3}, u_{3}\right) \\
& \left.+s_{3} ! \delta_{s_{2}}^{(1)} \phi_{s_{3}}\left(x_{3}, \partial_{u_{3}}\right) \hat{T}_{3} \partial_{x_{3}}^{2} \phi_{s_{3}}\left(x_{3}, u_{3}\right)\right]_{\substack{u_{i}=0 \\
x_{i}=x}}=0 .
\end{aligned}
$$

Employing (2.6) we get

$$
\begin{aligned}
\int d^{d} x[ & \left(s_{3} ! \delta_{s_{2}}^{(1)} \phi_{s_{3}}\left(x_{3}, \partial_{u_{3}}\right)-g_{0 s_{2} s_{3}} s_{2}\left(\partial_{u_{2}} \cdot \partial_{x_{31}}\right)^{s_{2}-1}\left(\partial_{u_{3}} \cdot \partial_{x_{12}}\right)^{s_{3}} \hat{T}_{3}^{-1} \phi_{0}\left(x_{1}\right) \varepsilon_{s_{2}-1}\left(x_{2}, u_{2}\right)\right) \\
& \left.\times \hat{T}_{3} \partial_{x_{3}}^{2} \phi_{s_{3}}\left(x_{3}, u_{3}\right)\right]_{\substack{u_{i}=0 \\
x_{i}=x}}=0 .
\end{aligned}
$$

After some simple manipulations this yields

$$
\begin{aligned}
\delta_{s_{2}}^{(1)} \phi_{s_{3}}\left(x_{3}, u_{3}\right)=g_{0 s_{2} s_{3}} \frac{s_{2}}{s_{3} !}[\{ & \left\{\hat{T}_{3}^{-1}\left(u_{3} \cdot \partial_{x_{12}}\right)^{s_{3}}\left(\partial_{u_{2}} \cdot\left(-2 \partial_{x_{1}}-\partial_{x_{2}}\right)\right)^{s_{2}-1}\right. \\
& \left.\left.\left.\times \phi_{0}\left(x_{1}\right) \varepsilon_{s_{2}-1}\left(x_{2}, u_{2}\right)\right\}\right\}\right]_{x_{1}=x_{2}=x_{3}},
\end{aligned}
$$

\footnotetext{
${ }^{13}$ Let us also mention that instead of solving a full-fledged version of (3.6) one may study a simpler consequence of it: one may consider deformations of gauge transformations induced by a cubic vertex and demand that they satisfy a generalised version of the Jacobi identity [4, 72]. In higher-spin theory in AdS it was shown $[65,66]$ that under some natural assumptions the general solution to the Jacobi identity is quite constraining. Then similarly to (3.7) this should allow one to express all non-Abelian cubic couplings in terms of a single coupling constant $\ell$, as was shown in the light-cone gauge in $[6,7]$.

${ }^{14}$ Deformations of gauge transformations induced by cubic couplings of higher spin fields and the associated gauge algebra deformations have been studied, in particular, in [4, 73-76]. However, due to certain issues (such as possible non-trivial contributions from non traceless-transverse terms, ambiguity in field redefinitions, etc.) these results cannot be directly used here.
} 
where $\{\{\ldots\}\}$ denotes the double-traceless projection in $u_{3}$, which in (3.10) was enforced implicitly by contraction with double-traceless $\phi_{s_{3}}$.

The presence of $\partial_{x_{1}}^{2}$ in (3.5) indicates that the cubic vertex (3.3) also induces a nontrivial transformation of the spin 0 field (which was not transforming at the leading order in (2.8)) with respect to the gauge symmetries of the higher spin fields. The explicit knowledge of this transformation will not, however, be required for the computation of the one-loop scalar self-energy below.

Let us remark on an ambiguity in the cubic vertex related to field redefinition freedom. The perturbative deformation procedure of the free gauge theory always has an ambiguity of additional local field redefinitions. Some preferred choice of basic fields may be selected by the requirement of the most simple form of the full non-linear theory consistent with manifest symmetries (cf. Einstein theory). Here we shall choose the "minimal" form (3.3) of the cubic vertex which is universal for all spins. When solving the gauge invariance condition (3.1) one usually ignores cubic vertices that vanish on the free equations of motion. Such vertices are "fake" in the sense that they can be generated from the free theory Lagrangian by field redefinitions. For example, in addition to the non-trivial vertex (3.3) one may consider, e.g., for $s_{2}=s_{3}=s$

$$
S^{(3)}\left[\phi_{0}, \phi_{s}, \phi_{s}\right] \sim \int d^{d} x \phi_{0}(x) \mathcal{R}_{s}(x) \cdot \mathcal{R}_{s}(x),
$$

where $\mathcal{R}_{s}$ is the de Wit-Freedman curvature [77] which is manifestly invariant under the linearized gauge transformations (2.8) (and thus does not induce any gauge symmetry deformation, i.e. $\delta^{(1)} \phi_{s}=0$ ). This does not contradict (3.11) implying that the deformation of the gauge symmetry is non-zero for $s_{2}=s_{3}$. Indeed, one can show that (3.11) for $s_{2}=s_{3}$ can be removed by a field redefinition. ${ }^{15}$

The local field redefinitions that preserve the asymptotic states should not, of course, change the on-shell amplitudes. That means that the "contact" contribution of the "oneshell trivial" 3-point vertex containing the free kinetic operator acting on one of the legs (i.e. the one that can be removed by a field redefinition) to, e.g., the 4-point scattering amplitude will be cancelled by the contribution of the 4 -vertex produced by the field redefinition.

\subsection{Ghost action}

According to the standard Faddeev-Popov procedure, the ghost action for the free theory (1.1), (2.11) invariant under (2.8) in the de Donder gauge (2.10) is

$$
\begin{aligned}
S^{(2)}\left[\bar{c}_{s-1}, c_{s-1}\right] & =(s-1) ! \int d^{d} x\left[\bar{c}_{s-1}\left(x, \partial_{u}\right) \hat{D} \frac{\delta_{s}^{(0)} \phi_{s}}{\delta \varepsilon_{s-1}} c_{s-1}(x, u)\right]_{u=0} \\
& =(s-1) ! \int d^{d} x\left[\bar{c}_{s-1}\left(x, \partial_{u}\right) \partial_{x}^{2} c_{s-1}(x, u)\right]_{u=0} .
\end{aligned}
$$

\footnotetext{
${ }^{15}$ For different spins $s_{2}<s_{3}$ the deformation of the gauge symmetry of the spin $s_{3}$ field is always nontrivial $[75,76]$ and the vertex cannot be put into the form (3.12).
} 
The cubic vertex involving the ghost fields is found analogously by using the deformation of the gauge transformation (3.11) instead of the leading-order transformation $\delta_{s}^{(0)} \phi_{s}$ in (3.13)

$$
\begin{aligned}
& S^{(3)}\left[\bar{c}_{s_{3}-1}, c_{s_{2}-1}, \phi_{0}\right] \\
&=\left(s_{3}-1\right) ! \int d^{d} x\left[\bar{c}_{s_{3}-1}\left(x_{3}, \partial_{u_{3}}\right) \hat{D}_{3} \frac{\delta_{s_{2}}^{(1)} \phi_{s_{3}}}{\delta \varepsilon_{s_{2}-1}} c_{s_{2}-1}\left(x_{3}, u_{3}\right)\right]_{u_{3}=0} \\
&=-g_{0 s_{2} s_{3}} \frac{s_{2}}{s_{3}} \int d^{d} x\left[\bar{c}_{s_{3}-1}\left(x_{3}, \partial_{u_{3}}\right)\left(\left(\partial_{x_{1}}+\partial_{x_{2}}\right) \cdot \partial_{u_{3}}-\frac{1}{2} u_{3} \cdot\left(\partial_{x_{1}}+\partial_{x_{2}}\right) \partial_{u_{3}}^{2}\right)\right. \\
&\left.\times\left\{\left\{\hat{T}_{3}^{-1}\left(u_{3} \cdot \partial_{x_{12}}\right)^{s_{3}}\left(\partial_{u_{2}} \cdot\left(-2 \partial_{x_{1}}-\partial_{x_{2}}\right)\right)^{s_{2}-1} \phi_{0}\left(x_{1}\right) c_{s_{2}-1}\left(x_{2}, u_{2}\right)\right\}\right\}\right]_{\substack{u_{i}=0 \\
x_{i}=x}} .
\end{aligned}
$$

The double-traceless projector in $u_{3}$ can be dropped as it is already imposed by the contraction with the remaining part of the integrand. After some straightforward algebra eq. (3.14) acquires a remarkably simple form

$$
\begin{aligned}
S^{(3)}\left[\phi_{0}, \bar{c}_{s_{3}-1}, c_{s_{2}-1}\right]=-g_{0 s_{2} s_{3}} s_{2} \int d^{d} x[ & \left(\partial_{u_{3}} \cdot \partial_{x_{12}}\right)^{s_{3}-1}\left(\partial_{u_{2}} \cdot \partial_{x_{31}}\right)^{s_{2}-1}\left(\partial_{x_{12}} \cdot \partial_{x_{3}}\right) \\
& \left.\times \bar{c}_{s_{3}-1}\left(x_{3}, u_{3}\right) \phi_{0}\left(x_{1}\right) c_{s_{2}-1}\left(x_{2}, u_{2}\right)\right]_{\substack{u_{i}=0 \\
x_{i}=x}} .
\end{aligned}
$$

\section{Feynman rules}

The propagator for the free Fronsdal field in the de Donder gauge was originally found in $d=4$ in [71] and was later extended to any dimension $d$ in [78]. For completeness, we shall review its derivation below. The expression for the propagator in terms of the Gegenbauer (or Chebyshev in $d=4$ ) polynomials was given in [79] that we follow here.

It is convenient to split the double-traceless field $\phi_{s}$ into two traceless fields $\nu_{s}$ and $\mu_{s-2}$ as

$$
\phi_{s}(x, u)=\nu_{s}(x, u)+\frac{1}{s(s-1)} u^{2} \mu_{s-2}(x, u) .
$$

Then the gauge fixed action (2.11) becomes

$$
\begin{aligned}
S^{(2)}\left[\phi_{s}\right]= & \frac{s !}{2} \int d^{d} x\left[\nu_{s}\left(x, \partial_{u}\right) \partial_{x}^{2} \nu_{s}(x, u)\right]_{u=0} \\
& -\frac{(d+2 s-4)(d+2 s-6)}{s(s-1)} \frac{(s-2) !}{2} \int d^{d} x\left[\mu_{s-2}\left(x, \partial_{u}\right) \partial_{x}^{2} \mu_{s-2}(x, u)\right]_{u=0} .
\end{aligned}
$$

The Fronsdal field propagator is found to be

$$
\mathcal{D}_{s}^{d}\left(u, u^{\prime} ; p\right)=-\frac{i}{p^{2}}\left[\mathcal{P}_{s}^{d}\left(u, u^{\prime}\right)+\frac{s(s-1)}{(d+2 s-4)(d+2 s-6)} \frac{u^{2} u^{\prime 2}}{s^{2}(s-1)^{2}} \mathcal{P}_{s-2}^{d}\left(u, u^{\prime}\right)\right]
$$

where $\mathcal{P}_{s}^{d}$ is a generating function for the standard rank- $s$ traceless projector bi-tensor

$$
\mathcal{P}_{s}^{d}\left(u, u^{\prime}\right)=\frac{1}{(s !)^{2}}\left(u \cdot u^{\prime}\right)^{s}+\ldots, \quad \partial_{u}^{2} \mathcal{P}_{s}^{d}\left(u, u^{\prime}\right)=\partial_{u^{\prime}}^{2} \mathcal{P}_{s}^{d}\left(u, u^{\prime}\right)=0
$$


It can be defined as a series

$$
\mathcal{P}_{s}^{d}\left(u, u^{\prime}\right)=\frac{1}{(s !)^{2}} \sum_{k=0}^{[s / 2]} t_{s, k}^{d}\left(u^{2}\right)^{k}\left(u^{\prime 2}\right)^{k}\left(u \cdot u^{\prime}\right)^{s-2 k},
$$

where

$$
t_{s, k}^{d}=\frac{(-1)^{k} s !}{4^{k} k !(s-2 k) !\left(\frac{d}{2}-1+s-k\right)_{k}}
$$

and $(a)_{k}=\Gamma(a+k) / \Gamma(a)$ is the Pochhammer symbol. It is convenient to rewrite (4.5) as

$$
\mathcal{P}_{s}^{d}\left(u, u^{\prime}\right)=\frac{1}{(s !)^{2}} \frac{s !}{\left(\frac{d}{2}-1\right)_{s}}\left(\frac{1}{2} \sqrt{u^{2} u^{\prime 2}}\right)^{s} C_{s}^{\frac{d}{2}-1}\left(\frac{u \cdot u^{\prime}}{\sqrt{u^{2} u^{\prime 2}}}\right)
$$

where

$$
C_{s}^{\alpha}(z) \equiv \sum_{k=0}^{[s / 2]} \frac{(-1)^{k}(\alpha)_{s-k}}{k !(s-2 k) !}(2 z)^{s-2 k}
$$

is the Gegenbauer polynomial. Observing that the coefficients in (4.6) satisfy

$$
t_{s, k}^{d}-\frac{s(s-1)}{(d+2 s-4)(d+2 s-6)} t_{s-2, k-1}^{d}=t_{s, k}^{d-2},
$$

or using the identity

$$
C_{s}^{\alpha}(z)-C_{s-2}^{\alpha}(z)=\frac{\alpha+s-1}{\alpha-1} C_{s}^{\alpha-1}(z),
$$

the propagator (4.3) can be put into the following simple form

$$
\mathcal{D}_{s}^{d}\left(u, u^{\prime} ; p\right)=-\frac{i}{p^{2}} \mathcal{P}_{s}^{d-2}\left(u, u^{\prime}\right) \text {. }
$$

Thus, in agreement with the result of [78], the tensorial part of the Fronsdal propagator is just the traceless projector in $d-2$ dimensions.

Let us note that for $d=4$ the higher spin propagator contains the two-dimensional projector $\mathcal{P}_{s}^{2}$, for which the representation in terms of the Gegenbauer polynomial (4.7) is singular due to vanishing of the denominator in the prefactor. Instead, one can use the expression

$$
\mathcal{P}_{s}^{2}\left(u, u^{\prime}\right)=\frac{1}{(s !)^{2}} 2\left(\frac{1}{2} \sqrt{u^{2} u^{\prime 2}}\right)^{s} T_{s}\left(\frac{u \cdot u^{\prime}}{\sqrt{u^{2} u^{\prime 2}}}\right)
$$

where

$$
T_{s}(z) \equiv \frac{s}{2} \sum_{k=0}^{[s / 2]} \frac{(-1)^{k}(s-k-1) !}{k !(s-2 k) !}(2 z)^{s-2 k}
$$

is the Chebyshev polynomial of the first kind. It can also be defined by

$$
T_{s}(z)=\frac{1}{2}\left[\left(z+\sqrt{z^{2}-1}\right)^{s}+\left(z-\sqrt{z^{2}-1}\right)^{s}\right] .
$$

The free ghost field in (3.13) is canonically normalised and traceless, i.e. has propagator

$$
\mathcal{G}_{s-1}^{d}\left(u, u^{\prime} ; p\right)=-\frac{i}{p^{2}} \mathcal{P}_{s-1}^{d}\left(u, u^{\prime}\right) .
$$


The cubic vertex for physical fields can be easily obtained from (3.3)

$$
\mathcal{V}\left(\partial_{u_{2}}, \partial_{u_{3}} ; p_{1}, p_{2}, p_{3}\right)=2 i g_{0 s_{2} s_{3}}\left(-i p_{31} \cdot \partial_{u_{2}}\right)^{s_{2}}\left(-i p_{12} \cdot \partial_{u_{3}}\right)^{s_{3}}
$$

where $p_{i j} \equiv p_{i}-p_{j}$ and 2 is a symmetry factor. ${ }^{16}$ This vertex is non-trivial only when $s_{2}+s_{3}$ is even (A.20). Similarly, for the ghost vertex we find from (3.15)

$$
\mathcal{W}\left(\partial_{u_{2}}, \partial_{u_{3}} ; p_{1}, p_{2}, p_{3}\right)=i g_{0 s_{2} s_{3}} s_{2}\left(-i p_{31} \cdot \partial_{u_{2}}\right)^{s_{2}-1}\left(-i p_{12} \cdot \partial_{u_{3}}\right)^{s_{3}-1}\left(-p_{12} \cdot p_{3}\right) .
$$

The resulting set of Feynman rules for the physical fields (wavy line or solid line for $s=0)$ and the ghosts (dotted line) can be summarised as follows

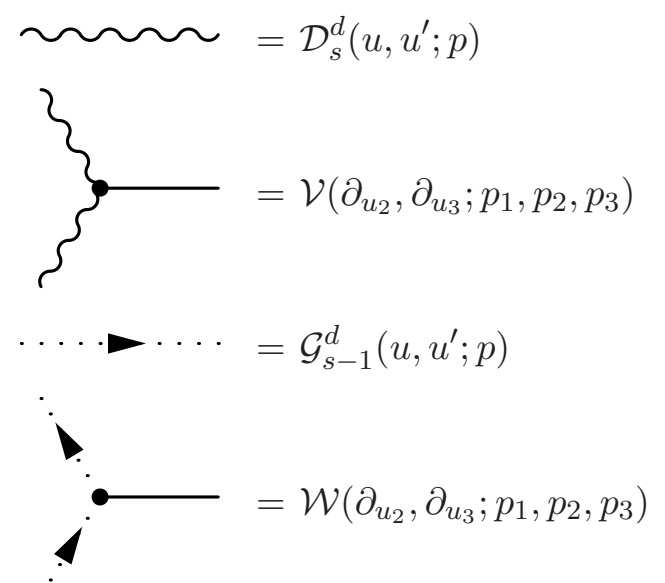

where explicit expressions are given in (4.7), (4.12), (4.11), (4.15)-(4.17) and the momenta are assumed to be ingoing.

\section{$5 \quad$ Tree-level 4-scalar scattering amplitude}

In this section we shall compute the tree-level 4-scalar scattering amplitude due to exchange of the tower of higher spin fields. For charged external scalar particles and arbitrary coupling constants in the corresponding cubic vertex this was earlier discussed in [79]. Here we shall repeat the same computation in the case of a real scalar which is the $s=0$ member of the tower of higher spin fields using the specific values of the cubic couplings given by $(3.7) \cdot{ }^{17}$

The important difference between the present case of a real scalar scattering and a complex scalar one in [79] is that in the real scalar case all interactions with odd spins vanish (cf. (3.3)). In the complex scalar case the odd spins contribute to the exchange

\footnotetext{
${ }^{16}$ Such symmetry factor appears when the vertex contains two identical fields. In the considered case spins $s_{2}$ and $s_{3}$ may be different. However, eventually, we are going to sum over spins and both $\phi_{s_{2}}$ and $\phi_{s_{3}}$ appear as particular members of two identical higher spin multiplets. Alternatively, this factor can be understood by arguing that during summation over spins each unordered pair $\left(s_{2}, s_{3}\right)$ is counted twice.

${ }^{17}$ While our final expression may be viewed as a special case of the general one in [79] the use of the particular values of the couplings in (3.7) is important as it leads to a convergent sum over all higher spin contributions and thus to a completely explicit expression for the exchange amplitude.
} 
amplitude with the opposite sign compared to the even spins. The resulting sum over spins contains terms with alternating signs which leads to an improved high energy behaviour [79] compared to the real scalar scattering case, where all exchange contributions add up with the same sign. ${ }^{18}$ Note that this remark applies to the full amplitude assuming the "contact" 4-point vertex contribution does not alter the UV asymptotics of the amplitude which need not be the case here (see discussion below).

\subsection{Exchange contribution}

The s-channel exchange of spin $j$ field gives (see (4.18))

$$
\sim\left\{\mathcal{A}_{\mathrm{exch}}^{j}(\mathrm{~s}, \mathrm{t}, \mathrm{u})=\mathcal{V}\left(\partial_{u} ; p_{1}, p_{2}, p\right) \mathcal{V}\left(\partial_{u^{\prime}} ; p_{1}^{\prime}, p_{2}^{\prime}, p\right) \mathcal{D}_{j}^{d}\left(u, u^{\prime} ; p\right),\right.
$$

where all the external momenta $p_{1}, p_{2}, p_{1}^{\prime}$ and $p_{2}^{\prime}$ are assumed to be ingoing: $p_{1}$ and $p_{2}$ into the left vertex and $p_{1}^{\prime}$ and $p_{2}^{\prime}$ into the right one $\left(p=p_{1}+p_{2}=-p_{1}^{\prime}-p_{2}^{\prime}\right)$. Let us introduce the Mandelstam variables

$\mathrm{s} \equiv-\left(p_{1}+p_{2}\right)^{2}, \quad \mathrm{t} \equiv-\left(p_{1}+p_{1}^{\prime}\right)^{2}, \quad \mathrm{u} \equiv-\left(p_{1}+p_{2}^{\prime}\right)^{2}, \quad \mathrm{~s}+\mathrm{t}+\mathrm{u}=0, \quad p_{i}^{2}=p_{i}^{\prime 2}=0$.

Using (4.11), (4.16) and that $p_{12}^{2}=p_{12}^{\prime 2}=-\mathrm{t}-\mathrm{u}, p_{12} \cdot p_{12}^{\prime}=-\mathrm{t}+\mathrm{u}$ we find ${ }^{19}$

$$
\mathcal{A}_{\text {exch }}^{j}(\mathrm{~s}, \mathrm{t}, \mathrm{u})=-\frac{i g_{00 j}^{2}}{\mathrm{~s}} \frac{2^{-j} j !}{\left(\frac{d}{2}-2\right)_{j}}(t+u)^{j} C_{j}^{\frac{d}{2}-2}\left(\frac{\mathrm{t}-\mathrm{u}}{\mathrm{t}+\mathrm{u}}\right) .
$$

In $d=4$ for an individual spin $j$ contribution we obtain

$$
\mathcal{A}_{\mathrm{exch}}^{j}(\mathrm{~s}, \mathrm{t}, \mathrm{u})=-\frac{i g_{00 j}^{2}}{\mathrm{~s}} 2^{-j+1}(\mathrm{t}+\mathrm{u})^{j} T_{j}\left(\frac{\mathrm{t}-\mathrm{u}}{\mathrm{t}+\mathrm{u}}\right)
$$

where $T_{j}(z)$ is given in (4.13), (4.14).

Summing over all even spins $j=2 k$ we obtain in $d=4$

$$
\mathcal{A}_{\text {exch }}(\mathrm{s}, \mathrm{t}, \mathrm{u})=\sum_{j=0,2,4, \ldots}^{\infty} \mathcal{A}_{\text {exch }}^{j}(\mathrm{~s}, \mathrm{t})=-\frac{i}{\mathrm{~s}} \sum_{k=0}^{\infty} g_{002 k}^{2} 2^{-2 k+1}(\mathrm{t}+\mathrm{u})^{2 k} T_{2 k}\left(\frac{\mathrm{t}-\mathrm{u}}{\mathrm{t}+\mathrm{u}}\right) \text {. }
$$

Let us introduce the function

$$
F(z) \equiv \sum_{k=0}^{\infty} g_{002 k}^{2} z^{2 k}=g^{2} \sum_{k=0}^{\infty} \frac{1}{[(2 k-1) !]^{2}}\left(\ell^{2} z\right)^{2 k},
$$

where we used the values of the couplings in (3.7) (note that here the $k=0$ term vanishes). It can be expressed in terms of the Bessel and the modified Bessel functions

$$
\begin{aligned}
& J_{\alpha}(z)=\left(\frac{z}{2}\right)^{\alpha} \sum_{k=0}^{\infty} \frac{(-1)^{k}}{\Gamma(k+1) \Gamma(\alpha+1+k)}\left(\frac{z}{2}\right)^{2 k}, \\
& I_{\alpha}(z)=\left(\frac{z}{2}\right)^{\alpha} \sum_{k=0}^{\infty} \frac{1}{\Gamma(k+1) \Gamma(\alpha+1+k)}\left(\frac{z}{2}\right)^{2 k}
\end{aligned}
$$

\footnotetext{
${ }^{18}$ Similar softening mechanism was discussed, e.g., in [80].

${ }^{19}$ Here the symmetry factor 2 in the cubic vertices (4.16) is not needed.
} 
as follows

$$
F(z)=\frac{1}{2} g^{2} \ell^{2} z\left(I_{0}(2 \ell \sqrt{z})-J_{0}(2 \ell \sqrt{z})\right) .
$$

Using (4.14) the s-channel amplitude (5.5) in $d=4$ may then be written as ${ }^{20}$

$$
\mathcal{A}_{\text {exch }}(\mathrm{s}, \mathrm{t}, \mathrm{u})=-\frac{i}{\mathrm{~s}}\left[F\left(\frac{1}{2}(\sqrt{\mathrm{s}+\mathrm{t}}+\sqrt{\mathrm{t}})^{2}\right)+F\left(\frac{1}{2}(\sqrt{\mathrm{s}+\mathrm{t}}-\sqrt{\mathrm{t}})^{2}\right)\right] .
$$

Note that for $\mathrm{s} \rightarrow 0$ the $\frac{1}{\mathrm{~s}}$ pole is not cancelled

$$
\mathrm{s} \rightarrow 0: \quad \mathcal{A}_{\text {exch }}(\mathrm{s}, \mathrm{t}, \mathrm{u})=-\frac{i}{\mathrm{~s}}\left[g^{2} \ell^{2} \mathrm{t}\left(I_{0}(2 \ell \sqrt{2 \mathrm{t}})-J_{0}(2 \ell \sqrt{2 \mathrm{t}})\right)+\mathcal{O}(\mathrm{s})\right] .
$$

The full exchange amplitude is found by adding also the contributions of the $t$ and $u$ channels,

$$
\hat{\mathcal{A}}_{\text {exch }}(\mathrm{s}, \mathrm{t}, \mathrm{u})=\mathcal{A}_{\text {exch }}(\mathrm{s}, \mathrm{t}, \mathrm{u})+\mathcal{A}_{\text {exch }}(\mathrm{t}, \mathrm{s}, \mathrm{u})+\mathcal{A}_{\text {exch }}(\mathrm{u}, \mathrm{t}, \mathrm{s}) .
$$

In the Regge limit ( $\mathrm{t} \rightarrow \infty$, s=fixed) the s-channel amplitude (5.9) gives the dominant contribution and using the standard asymptotics

$$
z \rightarrow \infty: \quad I_{\alpha}(z)=\frac{1}{\sqrt{2 \pi z}} e^{z}+\ldots, \quad J_{\alpha}(z)=\sqrt{\frac{2}{\pi z}} \cos \left(z-\frac{\alpha \pi}{2}-\frac{\pi}{4}\right)+\ldots,
$$

we find

$$
\mathrm{t} \rightarrow \infty, \mathrm{s}=\text { fixed }: \quad \hat{\mathcal{A}}_{\text {exch }}(\mathrm{s}, \mathrm{t}, \mathrm{u}) \sim-\frac{i g^{2}}{\mathrm{~s}} \ell^{2} \mathrm{t} I_{0}(2 \ell \sqrt{2 \mathrm{t}}) \sim-\frac{i g^{2}}{\mathrm{~s}} \frac{\left(\ell^{2} \mathrm{t}\right)^{3 / 4}}{2^{5 / 4} \pi^{1 / 2}} e^{2 \ell \sqrt{2 \mathrm{t}}}
$$

Similar behaviour is found also in the fixed-angle limit (cf. [79]). Analogous are expected also for other spin $s$ scattering amplitudes. Such an exponential growth of the tree-level scattering amplitude in the high energy regime is an indication of the presence of similar ultraviolet divergences in loop diagrams. Indeed, we shall find similar UV divergences in the one-loop self-energy bubble diagram contribution discussed in the next section.

Let us note that despite $\sqrt{\mathrm{s}}$ and $\sqrt{\mathrm{t}}$ arguments appearing in (5.9) the exchange amplitude is actually analytic in s, t. Indeed, the Bessel functions in (5.8) have expansion in even powers of their arguments and, as a consequence, the sum of the two $F$-functions in (5.9) has a convergent expansion in integer powers of $\mathrm{s}$ and $\mathrm{t}$ only. The appearance of a non-analytic function $e^{a \sqrt{t}}$ in (5.13) is just an artifact of the large $t$ limit. ${ }^{21}$

\subsection{Comments on 4-vertex contribution}

The full tree-level 4-point spin 0 scattering amplitude should also include the contribution of the 4-point 0-0-0-0 vertex:

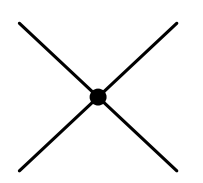

\footnotetext{
${ }^{20}$ In contrast to a similar amplitude in conformal higher-spin theory [27] here the sum over spins is convergent and the amplitude has non-trivial (non-scale-invariant) dependence on the Mandelstam variables due to the presence of the dimensionful parameter $\ell$.

${ }^{21}$ To give a simple example, $\cosh \sqrt{z}$ is an analytic function on the $z$-plane (given by a series of integer powers of $z$ ) but its large $z$ asymptotics is the same as that of a non-analytic function $\frac{1}{2} e^{\sqrt{z}}$.
} 
This vertex is expected to be effectively non-local (i.e. may contain an infinite series of powers of derivatives) and may thus "soften" the large momentum behaviour of the exchange diagram contribution.

While the precise form of the 4-scalar vertex in the flat-space action (1.1) is presently unknown, we may try to get some idea about its structure using an analogy with a similar term in the action (1.2) of the massless higher-spin theory in AdS. The 0-0-0-0 term in the AdS action was recently reconstructed [52] from the free boundary CFT data by assuming the AdS/CFT correspondence. We shall use a heuristic trick of replacing the AdS covariant derivatives by the flat space ones in the expression in [52]. This then gives

$$
S^{(4)}\left[\phi_{0}\right]=g^{2} \int d^{4} x\left[\sum_{j=0}^{\infty} f_{2 j}\left(\Delta_{x_{34}}\right)\left(\partial_{x_{12}} \cdot \partial_{x_{34}}\right)^{2 j} \phi_{0}\left(x_{1}\right) \phi_{0}\left(x_{2}\right) \phi_{0}\left(x_{3}\right) \phi_{0}\left(x_{4}\right)\right]_{x_{i}=x}
$$

where $\Delta_{x_{34}} \equiv\left(\partial_{x_{3}}+\partial_{x_{4}}\right)^{2}, \partial_{x_{12}} \equiv \partial_{x_{1}}-\partial_{x_{2}}$ and the function $f_{2 j}(z)$ is given by some infinite power series in $z$. To estimate the large momentum expansion of the resulting amplitude we may choose the large $z$ asymptotics of the function $f_{2 j}(z)$ to be the same as in corresponding function in the AdS 4-scalar vertex in [52], i.e.

$$
f_{2 j}(z)=c_{2 j} \frac{\ell^{4 j-2}}{z}, \quad z \rightarrow \infty
$$

Here $c_{2 j}$ are numerical coefficients and we introduced the parameter $\ell$ to balance the dimensions. ${ }^{22}$ Given $(5.14),(5.15)$ the contribution of the 4 -vertex to the 4 -scalar amplitude may, in principle, cancel the exponential growth (5.13) of the exchange amplitude. For example, choosing $c_{2 j}$ in the form similar to the one they have in the AdS expression [52]

$$
c_{2 j}=\frac{1}{[(2 j-1) !]^{2}},
$$

we find that the asymptotic contribution of the vertex (5.14) to the 4-point amplitude is proportional to

$$
\begin{aligned}
\sum_{j=0}^{\infty} f_{2 j}\left(-\left(p_{3}+p_{4}\right)^{2}\right)\left[\left(p_{1}-p_{2}\right) \cdot\left(p_{3}-p_{4}\right)\right]^{2 j} & =\sum_{j=0}^{\infty} f_{2 j}(\mathrm{~s})(\mathrm{t}-\mathrm{u})^{2 j} \\
& =\frac{2 \mathrm{t}+\mathrm{s}}{2 \mathrm{~s}}\left[I_{0}(2 \ell \sqrt{2 \mathrm{t}+\mathrm{s}})-J_{0}(2 \ell \sqrt{2 \mathrm{t}+\mathrm{s}})\right]
\end{aligned}
$$

where $\mathrm{s}$ is assumed to be large. It is thus also expressed in terms of the Bessel functions as in (5.8), (5.9), i.e. has a similar Regge behaviour as in (5.13).

In the case of higher-spin theory in AdS one may interpret the result for the tree-level amplitude (that reproduces the free CFT 4-point correlator) as indicating a soft behaviour in the UV. It was previously observed that the expressions for the Witten diagrams in AdS written in the Mellin representation look similar to the scattering amplitudes for the

\footnotetext{
${ }^{22}$ Note that the exact expression for $f_{2 j}(z)$ in [52] does not have poles (in particular, is regular at $z \rightarrow 0$ ), so its flat-space counterpart should also not contain an essential non-locality like $\frac{1}{\partial^{2}}$.
} 
same processes in flat space [58]. In particular, the contact interactions with $2 n$ derivatives give rise to the Mellin amplitudes given by polynomials of $n$-th degree in the Mellin variables (which play the role of the AdS counterparts of the Mandelstam variables). The AdS exchanges produce the Mellin amplitudes with poles associated to the dimensions of the exchanged operators and their descendants. The total four point scattering amplitude should have a similar structure to the Mellin amplitude for the 4-point correlator of spin 0 primary operator in the free $O(N)$ CFT. This amplitude is a distribution [81, 82] in the sense that it is a certain combination of delta functions and hence it is zero everywhere except certain specific values of the Mellin variables. That could be interpreted as suggesting that the total tree-level 4-scalar amplitude in flat space may also be a distribution and thus should have trivial UV asymptotics. ${ }^{23}$ It is not clear, however, how such simple amplitude could come out of an explicit flat-space scattering computation (for example, adding the 4 -vertex contribution is unlikely to cancel the massless pole appearing in the exchange contribution).

Finally, let us note that the cubic vertices (3.7) appear to be inconsistent with the BCFW constructibility condition. This was previously discussed in a similar context in [10, 69]. This condition requires that the scattering amplitudes vanish under infinite complex shifts of momenta [21]. Together with the assumption of analyticity ${ }^{24}$ this leads to certain recurrence relations which allow one to express any tree-level amplitude in terms of the on-shell three-point ones. Applied to the four-scalar amplitude that we have studied above this would allow one to determine the quartic scalar self-coupling in terms of the cubic vertices (3.3), (3.7). However, the BCFW construction can be applied only if the cubic vertices satisfy a certain non-trivial consistency condition, the so called the four-particle test [21]. As we will show in appendix B, the cubic vertices (3.3), (3.7) fail to satisfy this test.

It is not clear a priori if the condition of BCFW constructibility should, in fact, apply to an effectively non-local theory like (1.1) containing infinite number of higher-spin fields with higher derivatives of any order in vertices. For example, the BCFW approach relies on the assumption of the analyticity of the amplitudes. While perturbative amplitudes reconstructed from higher spin vertices will be given by sums of integer powers of momenta (i.e. are analytic before summation over spins) the sums over spins could not converge fast enough. This did not happen for the exchange amplitude (5.9) discussed above but was the case for the 4-scalar scattering in the conformal higher-spin theory [27] where the amplitude was not analytic — was given by a distribution.

\section{One-loop scalar self-energy correction}

Let us now consider the spin 0 one-loop self-energy correction. It is given by the sum of the two parts - of bubble diagrams and of tadpole diagrams. Each of these contains

\footnotetext{
${ }^{23}$ Similar behaviour was found for the external scalar scattering amplitude in the conformal higher-spin theory [27] (there the amplitude actually vanished for the physical values of the Mandelstam variables).

${ }^{24}$ If an analytic function vanishes at infinity it can be reconstructed from poles and their residues.
} 
contributions from the physical higher spin loops and from the ghost loops:

Bubble diagrams:

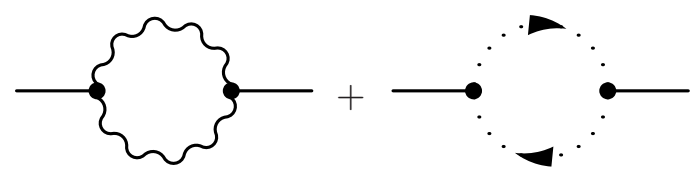

Tadpole diagrams:

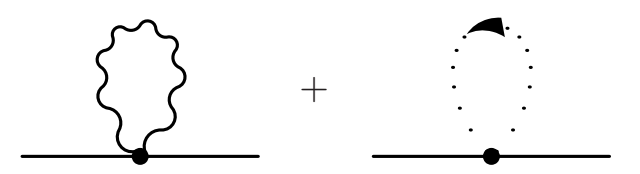

The bubble diagrams are straightforward to evaluate using the Feynman rules described in section 4. The tadpole diagrams contain the physical 4-point $0-0-s$-s vertices $S^{(4)}$ and their ghost counterparts (determined by subleading gauge symmetry deformations $\delta^{(2)} \phi$ quadratic in fields) which are not known at present. While we will not be able to compute the tadpole diagram, the bubble diagram already provides an important information about the self-energy contribution as it captures a non-trivial part of its dependence on external momenta. Indeed, the tadpole contribution is expected to be a regular function of external momentum while the bubble one should contain branch cuts coming from logarithmic terms appearing from massless loops.

\subsection{Individual bubble diagrams}

Let us start with the bubble diagram with two physical field propagators in the loop. The momenta of particles ingoing the left vertex will be denoted $p_{1}$ for the external scalar and $p_{2}$ and $p_{3}$ for the spin $s_{2}$ and spin $s_{3}$ fields. Similarly, the momenta ingoing the right vertex will be $p_{1}^{\prime}$ for the external scalar and $p_{2}^{\prime}$ and $p_{3}^{\prime}$ for higher-spin fields, i.e.

$$
p_{1}+p_{2}+p_{3}=0, \quad p_{1}^{\prime}+p_{2}^{\prime}+p_{3}^{\prime}=0, \quad p_{1}=-p_{1}^{\prime}, \quad p_{2}=-p_{2}^{\prime}, \quad p_{3}=-p_{3}^{\prime} .
$$

We shall also use the notation $k \equiv p_{1}$ for the external momentum and $p \equiv-p_{2}$ for the virtual momentum. The contribution of such bubble diagram reads ${ }^{25}$

$$
\begin{gathered}
\qquad\left\{=\frac{1}{2} \int d^{d} p_{2} \mathcal{V}\left(\partial_{u_{2}}, \partial_{u_{3}} ; p_{1}, p_{2}, p_{3}\right) \mathcal{V}\left(\partial_{u_{2}^{\prime}}, \partial_{u_{3}^{\prime}} ; p_{1}^{\prime}, p_{2}^{\prime}, p_{3}^{\prime}\right)\right. \\
\times \mathcal{D}_{s_{2}}^{d}\left(u_{2}, u_{2}^{\prime} ; p_{2}\right) \mathcal{D}_{s_{3}}^{d}\left(u_{3}, u_{3}^{\prime} ; p_{3}\right),
\end{gathered}
$$

where $\frac{1}{2}$ is a symmetry factor and $s_{2}, s_{3}$ may take any values including 0 . Here $\mathcal{V}$ is the cubic vertex from (3.3), (4.16) and $\mathcal{D}_{s}^{d}$ is the propagator from (4.11) (cf. (4.18)). The dependence on $u$-variables is spurious - it goes away after acting by derivatives $\partial_{u}$. For example, the left vertex $\mathcal{V}$ contains an operator $\left(-i p_{31} \cdot \partial_{u_{2}}\right)^{s_{2}}$ which should be applied to the propagator $\mathcal{D}_{s_{2}}$ thus performing the tensor index contraction. Given that $\mathcal{D}_{s_{2}}$ is a homogeneous polynomial of degree $s_{2}$ in $u_{2}$ (cf. (4.5), (4.8)) the result of this contraction

\footnotetext{
${ }^{25}$ We omit the standard overall factor $(2 \pi)^{-d}$ that should be reinstated in the final expression for the self-energy $\Sigma\left(k^{2}\right)$.
} 
amounts to replacing $u_{2} \rightarrow-i p_{31}$ inside the propagator and also cancelling the factor of $s_{2}$ ! in the denominator. Computing other contractions in the same way we find

$$
\widetilde{\{}=2 g_{0 s_{2} s_{3}}^{2} \int \frac{d^{d} p_{2}}{p_{2}^{2} p_{3}^{2}} \frac{s_{2} ! C_{s_{2}}^{\frac{d}{2}-2}(1)}{2^{s_{2}}\left(\frac{d}{2}-2\right)_{s_{2}}} \frac{s_{3} ! C_{s_{2}}^{\frac{d}{2}-2}(1)}{2^{s_{3}}\left(\frac{d}{2}-2\right)_{s_{3}}}\left(p_{31}^{2}\right)^{s_{2}}\left(p_{12}^{2}\right)^{s_{3}} \text {, }
$$

where $g_{0 s_{2} s_{3}}$ is the cubic coupling constant (to be chosen as in (3.7)) and $C_{s_{2}}^{\frac{d}{2}-2}(1)$ is found from (4.8), i.e.

$$
C_{s}^{\alpha}(1)=\frac{\Gamma(2 \alpha+s)}{\Gamma(2 \alpha) \Gamma(s+1)}
$$

We thus obtain ${ }^{26}$

$$
\{\}=2 g_{0 s_{2} s_{3}}^{2} \frac{(d-4)_{s_{2}}}{2^{s_{2}\left(\frac{d}{2}-2\right)_{s_{2}}}} \frac{(d-4)_{s_{3}}}{2^{s_{3}}\left(\frac{d}{2}-2\right)_{s_{3}}} \int \frac{d^{d} p_{2}}{p_{2}^{2} p_{3}^{2}}\left(p_{31}^{2}\right)^{s_{2}}\left(p_{12}^{2}\right)^{s_{3}} \text {. }
$$

The ghost loop contribution can be computed in a similar way using (4.15), (4.17), (4.18)

$$
\begin{aligned}
\because \because- & g_{0 s_{2} s_{3}}^{2} \frac{s_{2}(d-2)_{s_{2}-1}}{2^{s_{2}-1}\left(\frac{d}{2}-1\right)_{s_{2}-1}} \frac{s_{3}(d-2)_{s_{3}-1}}{2^{s_{3}-1}\left(\frac{d}{2}-1\right)_{s_{3}-1}} \\
& \times \int \frac{d^{d} p_{2}}{p_{2}^{2} p_{3}^{2}}\left(p_{31}^{2}\right)^{s_{2}-1}\left(p_{12}^{2}\right)^{s_{3}-1}\left(p_{31} \cdot p_{2}\right)\left(p_{3} \cdot p_{12}\right) .
\end{aligned}
$$

Combining (6.5) and (6.6) and expressing the momenta in terms of $k \equiv p_{1}$ and $p \equiv-p_{2}$ we get for the bubble diagram contribution with the spin $s_{2}, s_{3}$ loop

$$
\begin{aligned}
& \Sigma_{s_{2} s_{3}}\left(k^{2}\right)=g_{0 s_{2} s_{3}}^{2} \int \frac{d^{d} p}{p^{2}(p-k)^{2}}(2 k-p)^{2 s_{2}}(k+p)^{2 s_{3}}\left[2 \frac{(d-4)_{s_{2}}}{2^{s_{2}\left(\frac{d}{2}-2\right)_{s_{2}}}} \frac{(d-4)_{s_{3}}}{2^{s_{3}}\left(\frac{d}{2}-2\right)_{s_{3}}}\right. \\
& \left.+\frac{s_{2}(d-2)_{s_{2}-1}}{2^{s_{2}-1}\left(\frac{d}{2}-1\right)_{s_{2}-1}} \frac{s_{3}(d-2)_{s_{3}-1}}{2^{s_{3}-1}\left(\frac{d}{2}-1\right)_{s_{3}-1}} \frac{\left(2 k p-p^{2}\right)\left(p^{2}-k^{2}\right)}{(2 k-p)^{2}(k+p)^{2}}\right] .
\end{aligned}
$$

\subsection{Summing over spins}

Using the expression for the coupling constants in (3.7) and specifying to $d=4$ we find for the sum $\Sigma\left(k^{2}\right)$ of the individual contributions (6.7)

$$
\begin{aligned}
& \Sigma\left(k^{2}\right)=g^{2} \int \frac{d^{4} p}{p^{2}(p-k)^{2}} \sum_{\substack{s_{2}, s_{3}=0 \\
s_{2}+s_{3}=\text { even } \\
\\
+}}^{\infty} \frac{\ell^{2\left(s_{2}+s_{3}-1\right)}}{s^{s_{2}-1} s_{3}^{2}(2 k-p)^{s_{3}-1}\left[\left(s_{2}+s_{3}-1\right) !\right]^{2}}\left[2(2 k-p)^{2 s_{2}}(k+p)^{2 s_{3}}\right. \\
&
\end{aligned}
$$

\footnotetext{
${ }^{26}$ Note that the coefficient here is regular at $d=4: \frac{(d-4)_{s}}{\left(\frac{d}{2}-2\right)_{s}}=\frac{(d-4)(d-3)_{s-1}}{\left(\frac{d}{2}-2\right)\left(\frac{d}{2}-1\right)_{s-1}} \rightarrow 2$.
} 
Here the sum goes over even $s_{2}+s_{3}$ because the vertex (3.3) vanishes when the total spin $s_{2}+s_{3}$ is odd and the same is also true for the ghost vertex (3.15). ${ }^{27}$ Evaluating the sum one finds (see appendix $\mathrm{C}$ for details)

$$
\begin{aligned}
\Sigma\left(k^{2}\right)= & g^{2} \ell^{-2} \int \frac{d^{4} p}{p^{2}(p-k)^{2}} \Phi(p, k), \\
\Phi(p, k)= & \left(\frac{4 x^{2}}{x-y}+\frac{2\left(2 k p-p^{2}\right)\left(p^{2}-k^{2}\right)}{(2 k-p)^{2}(k+p)^{2}} \frac{4 x^{2} y^{2}\left(x^{2}+4 x y+y^{2}\right)}{(x-y)^{5}}\right)\left[I_{0}(2 \sqrt{x})-J_{0}(2 \sqrt{x})\right] \\
& +\frac{2\left(2 k p-p^{2}\right)\left(p^{2}-k^{2}\right)}{(2 k-p)^{2}(k+p)^{2}}\left(\frac{x^{3} y(x+y)}{(x-y)^{3}}\left[I_{2}(2 \sqrt{x})-J_{2}(2 \sqrt{x})\right]\right. \\
& \left.+\frac{2 x^{2} y\left(x^{2}-8 x y-5 y^{2}\right) \sqrt{x}}{(x-y)^{4}}\left[I_{1}(2 \sqrt{x})+J_{1}(2 \sqrt{x})\right]\right)+(x \leftrightarrow y),
\end{aligned}
$$

where $I_{n}$ and $J_{n}$ are the Bessel functions (5.7) and we defined

$$
x \equiv \frac{1}{2} \ell^{2}(2 k-p)^{2}, y \equiv \frac{1}{2} \ell^{2}(k+p)^{2} .
$$

\subsection{UV divergences and regularization}

Let us now discuss the UV divergence of $\Sigma$. There are at least two possible approaches: (i) first introduce an explicit momentum UV cutoff, sum over spins and then take cutoff to infinity, or (ii) first drop all power divergences in each individual loop using the dimensional regularization and then sum over spins. ${ }^{28}$

Following the first approach, to isolate the UV divergence of the loop integral in (6.10) let us consider the $p \rightarrow \infty, k=$ fixed limit of the integrand. In this limit $\frac{x}{y} \rightarrow 1$. To extract the leading singularity one may just set $k=0$. The apparent $x=y$ poles of the integrand are spurious and one finds (see appendix $\mathrm{C}$ )

$$
\begin{aligned}
\Sigma(0)=2 g^{2} \ell^{-2} \int \frac{d^{4} p}{p^{4}}( & -\frac{1}{30}\left(\frac{\ell p}{\sqrt{2}}\right)^{7}\left[I_{5}(\sqrt{2} \ell p)+J_{5}(\sqrt{2} \ell p)\right]-\frac{1}{2}\left(\frac{\ell p}{\sqrt{2}}\right)^{6}\left[I_{4}(\sqrt{2} \ell p)-J_{4}(\sqrt{2} \ell p)\right] \\
& -\frac{13}{6}\left(\frac{\ell p}{\sqrt{2}}\right)^{5}\left[I_{3}(\sqrt{2} \ell p)+J_{3}(\sqrt{2} \ell p)\right]-3\left(\frac{\ell p}{\sqrt{2}}\right)^{4}\left[I_{2}(\sqrt{2} \ell p)-J_{2}(\sqrt{2} \ell p)\right] \\
+ & \left.\left(\frac{\ell p}{\sqrt{2}}\right)^{3}\left[I_{1}(\sqrt{2} \ell p)+J_{1}(\sqrt{2} \ell p)\right]+4\left(\frac{\ell p}{\sqrt{2}}\right)^{2}\left[I_{0}(\sqrt{2} \ell p)-J_{0}(\sqrt{2} \ell p)\right]\right) .
\end{aligned}
$$

From the asymptotic behaviour of the Bessel functions (5.12) we conclude that $\Sigma$ in (6.9) is exponentially UV divergent, i.e. introducing an explicit UV momentum cutoff $\Lambda$ we get

$$
\Sigma(0) \sim g^{2} \ell^{-2} \int^{\Lambda} \frac{d^{4} p}{p^{4}}\left(\left[(\ell p)^{13 / 2}+\ldots\right] e^{\sqrt{2} \ell p}+\ldots\right) \sim g^{2} \ell^{-2}(\ell \Lambda)^{11 / 2} e^{\sqrt{2} \ell \Lambda}+\ldots
$$

\footnotetext{
${ }^{27}$ The cubic vertex $S\left[\phi_{0}, \phi_{s_{2}}, \phi_{s_{3}}\right]$ leads to the two terms in the ghost action, schematically $V_{1}=$ $S\left[\bar{c}_{s_{3}-1}, c_{s_{2}-1}, \phi_{0}\right]$ and $V_{2}=S\left[\bar{c}_{s_{2}-1}, c_{s_{3}-1}, \phi_{0}\right]$ (see (3.14)). Similarly, the vertex $S\left[\phi_{0}, \phi_{s_{3}}, \phi_{s_{2}}\right]$ leads to $V_{3}=S\left[\bar{c}_{s_{2}-1}, c_{s_{3}-1}, \phi_{0}\right]$ and $V_{4}=S\left[\bar{c}_{s_{3}-1}, c_{s_{2}-1}, \phi_{0}\right]$. One can check that $V_{1}=(-1)^{s_{2}+s_{3}} V_{4}$ and $V_{2}=(-1)^{s_{2}+s_{3}} V_{3}$, so that for $s_{2}+s_{3}=$ odd the ghost action vanishes.

${ }^{28}$ In general, dimensional regularization is known to be a preferable choice in order to preserve gauge invariance of the theory at the quantum level but in an effectively non-local theory like the present one its use needs to be further justified, e.g., it may not commute with summation over spins (see also below).
} 
Keeping also the subleading order $k^{2}$ term in the large $p$ expansion of the integrand in (6.9) gives

$$
\begin{aligned}
\Sigma\left(k^{2}\right)=g^{2} \ell^{-2} \int^{\Lambda} \frac{d^{4} p}{p^{4}}( & {\left[-\frac{1}{15}\left(\frac{\ell p}{\sqrt{2}}\right)^{7}+\frac{1}{15}\left(\frac{\ell p}{\sqrt{2}}\right)^{8} \frac{k p}{p^{2}}-\frac{8}{105}\left(\frac{\ell p}{\sqrt{2}}\right)^{9}\left(\frac{k p}{p^{2}}\right)^{2}\right.} \\
& \left.\left.\quad-\frac{1}{6}\left(\frac{\ell p}{\sqrt{2}}\right)^{8} \frac{k^{2}}{p^{2}}+\ldots\right] \frac{e^{\sqrt{2} \ell p}}{2^{3 / 4} \sqrt{\pi \ell p}}+\ldots\right) \\
\sim & g^{2} \ell^{-2}\left(1+\frac{1}{7} \ell^{2} k^{2}+\ldots\right)(\ell \Lambda)^{11 / 2} e^{\sqrt{2} \ell \Lambda}+\ldots
\end{aligned}
$$

Note also that the logarithmic $\log \frac{\Lambda}{k}$ divergence in (6.9), (6.14) has a non-zero coefficient

$$
\Phi(0, k)=\frac{2}{3} \ell^{2} k^{2}\left(16\left[I_{0}(2 \sqrt{2} \ell k)-J_{0}(2 \sqrt{2} \ell k)\right]-\left[I_{0}(\sqrt{2} \ell k)-J_{0}(\sqrt{2} \ell k)\right]\right) .
$$

Instead of using the UV momentum cutoff in the summed over spins expression one may first define each loop integral (6.7) with the help of dimensional regularization which gets rid of all power divergences, i.e. sets

$$
\int d^{4} p\left(p^{2}\right)^{n}=0, \quad n=-1,0,1, \ldots
$$

Then for integer $n=-1,0,1 \ldots$ and $m=0,1, \ldots$ (or vice versa) one has also

$$
\int d^{4} p\left(p^{2}\right)^{m}\left[(p-k)^{2}\right]^{n}=0
$$

The integral (6.8) has the general form

$$
\Sigma\left(k^{2}\right)=g^{2} \ell^{-2} \int \frac{d^{4} p}{p^{2}(p-k)^{2}} \Phi\left(p^{2},(p-k)^{2}, k^{2}\right),
$$

where the function $\Phi$ is a series in positive integer powers of its arguments. Then assuming (6.17) we conclude that only $\Phi\left(0,0, k^{2}\right) \equiv \Phi\left(k^{2}\right)$ produces a non-zero contribution in dimensional regularisation, i.e. we are left with only logarithmically divergent integral

$$
\Sigma\left(k^{2}\right)=g^{2} \ell^{-2} \Phi\left(k^{2}\right) \int \frac{d^{4} p}{p^{2}(k-p)^{2}}, \quad \Phi\left(k^{2}\right)=\sum_{\substack{s_{2}, s_{3}=0 \\ s_{2}+s_{3}=\text { even }}}^{\infty} \frac{\left(8-s_{2}^{2} s_{3}^{2}\right)\left(\ell^{2} k^{2}\right)^{s_{2}+s_{3}}}{\left[\left(s_{2}+s_{3}-1\right) !\right]^{2}} .
$$

Computing the sum as discussed in appendix $\mathrm{C}$ we get

$$
\begin{aligned}
\Phi\left(k^{2}\right)= & -\frac{1}{60}(\ell k)^{7}\left[I_{5}(2 \ell k)+J_{5}(2 \ell k)\right]-\frac{1}{4}(\ell k)^{6}\left[I_{4}(2 \ell k)-J_{4}(2 \ell k)\right] \\
& -\frac{13}{12}(\ell k)^{5}\left[I_{3}(2 \ell k)+J_{3}(2 \ell k)\right]-\frac{3}{2}(\ell k)^{4}\left[I_{2}(2 \ell k)-J_{2}(2 \ell k)\right] \\
& +\frac{7}{2}(\ell k)^{3}\left[I_{1}(2 \ell k)+J_{1}(2 \ell k)\right]+8(\ell k)^{2}\left[I_{0}(2 \ell k)-J_{0}(2 \ell k)\right] .
\end{aligned}
$$

Thus with this dimensional regularization prescription (where one discards all power divergences before summation over spins) one finds that instead of an exponential divergence 
in (6.13) the self-energy divergerges only logarithmically ${ }^{29}$ and, in particular, vanishes at zero momentum, $\Sigma(0)=0$, so that the spin 0 field remains massless.

\subsection{Comments on tadpole diagram contribution}

One may wonder if the exponential divergence (6.13) may get cancelled upon adding the tadpole diagram contribution $\Sigma_{\mathrm{tp}}\left(k^{2}\right)$. As we have seen in section 5 , the expected structure of the 4-vertex (5.14) leads to a contribution to the 4-scalar tree-level scattering amplitude that has similar exponential large momentum behaviour (5.17) as the exchange diagram (5.13). One may thus expect that this 4-vertex contribution to the self-energy tadpole diagram will also have an exponential UV behaviour similar to the one in (6.13).

Let us consider just a single virtual $s=0$ field contribution to the tadpole diagram for which the knowledge of the 0-0-0-0 vertex (5.14) is sufficient. Computing the scalar loop with two scalar external legs we then get the following estimate for its large virtual momentum behaviour (using (5.15), (5.16), cf. (5.17))

$$
\Sigma_{\mathrm{tp}}\left(k^{2}\right) \sim g^{2} \int \frac{d^{4} p}{p^{2}} \sum_{j=0}\left[f_{2 j}\left(-(k-p)^{2}\right)\left[(k+p)^{2}\right]^{2 j}+f_{2 j}\left(-(k+p)^{2}\right)\left[(k-p)^{2}\right]^{2 j}\right] .
$$

Setting $k=0$ to get the leading UV asymptotics we find

$$
\Sigma_{\mathrm{tp}}(0) \sim g^{2} \int \frac{d^{4} p}{p^{2}} \sum_{j=0} a_{2 j}\left(-p^{2}\right)\left(p^{2}\right)^{2 j}=\frac{1}{4} g^{2} \int \frac{d^{4} p}{p^{2}}\left[I_{0}(\sqrt{2} \ell p)-J_{0}(\sqrt{2} \ell p)\right] .
$$

Thus this tadpole diagram has a similar exponential UV behaviour as the bubble diagram (6.10).

This gives a hope that the UV divergence of the bubble diagram contribution may get cancelled once all tadpole diagrams (for all spins propagating in the loop) are taken into account. Ideally, the sum of the bubble and tadpole contributions may be given by a convergent momentum integral that will not require UV regularization and may actually vanish. ${ }^{30}$ The same may be true also for the case of self-energy diagram with an arbitrary spin $s$ field on external lines. Such a result is expected in the AdS higher-spin theory dual to a boundary $\mathrm{U}(N)$ or $O(N)$ scalar where a one-loop self-energy correction would represent a $1 / N$ correction to the 2-point function of conserved currents which should be absent in a free theory (i.e. a theory with unbroken higher spin symmetry).

\footnotetext{
${ }^{29}$ The self-energy diagram we are computing is an off-shell one, and as in scalar electrodynamics this logarithmic divergence may be absorbed into a wave function renormalization of the spin 0 field.

${ }^{30}$ The prescription where one first combines all contributions together, sums over spins and only then discusses the need for a UV regularization seems the most natural one. The application of dimensional regularization to individual graphs may be objected as it may not commute with summation over spins. For example, a convergent integral like $\int \frac{d^{4} p e^{-\ell^{2} p^{2}}}{p^{2}(k-p)^{2}}$ may be represented as a sum of divergent integrals $\sum_{n=0}^{\infty} \frac{(-1)^{n}}{n !} \int \frac{d^{4} p\left(\ell^{2} p^{2}\right)^{n}}{p^{2}(k-p)^{2}}$ with all $n \geq 1$ terms vanishing if computed in dimensional regularization but the remaining $n=0$ one having the logarithmic divergence.
} 


\section{Concluding remarks}

In this paper we have computed the one-loop bubble diagram correction to the scalar propagator generated by interactions with an infinite tower of higher-spin gauge fields in flat $4 \mathrm{~d}$ space. One motivation was to investigate whether in higher-spin theories, similarly to what happens in string theory, the summation over an infinite number of spins may make loop integrals finite in the ultraviolet. Another is that this may also be considered as a simplified version of the analogous computation in the massless AdS higher-spin theory, aiming to verify a remarkable prediction of the vectorial AdS/CFT duality that all loop corrections in this higher-spin theory should vanish.

The explicit cubic coupling coefficients (3.7) that we used were previously derived by demanding consistency of higher-spin interactions to the subleading $g^{2}$ order in the lightcone approach [6]. We also used these coefficients to compute the tree scattering amplitude of the massless scalars due to the exchange of an infinite tower of massless higher spins. We found that this exchange amplitude has an exponential growth in the Regge limit, suggesting singular UV behaviour in the loops. Indeed, the bubble diagram contribution to the scalar self-energy (summed over all virtual spins propagating in the loop) was found to be exponentially divergent at high energies.

Qualitatively, this happens because all contributions from different spins (each of which grows in the UV due to the presence of higher derivatives in the cubic vertex) enter with the same sign and thus the summation over spins cannot improve the ultraviolet behaviour. The external spin 0 field we were scattering is a member of the higher-spin tower, i.e. a real scalar which couples via $0-0-s$ vertex only to even spin $s$ fields. ${ }^{31}$

In addition to the exchange diagrams, the full tree-level 4-scalar amplitude should contain also the contribution of the 4-point vertex. Similarly, besides the bubble diagrams, the full one-loop self-energy correction should contain also the contributions of the tadpole diagrams. To compute these extra contributions requires the knowledge of quartic vertices which are not fully understood at present. To get an idea of possible structure of 4 -vertex contributions we used the 4-scalar interaction term found in the AdS higher-spin theory [52] and formally continued it to flat space in the short distance limit. We found that it leads, indeed, to similar UV behaviour as the tree-level exchange diagrams and also to similar tadpole momentum integrals as appear in the bubble diagram. There is thus a potential possibility of cancellation of UV divergences in the full one-loop self-energy correction. This is what is to happen in the AdS higher-spin theory dual to a boundary CFT and would be in line with the expectation that the UV behaviour in the flat space and AdS theories should be similar.

\footnotetext{
${ }^{31}$ At the same time, if one considers the scattering of a complex scalar that couples also to odd spins (with extra $i$ in the vertex) one may get softer UV behaviour due to alternating signs of coefficients in sum over all spins [79]. It is not clear, however, if there is a consistent higher-spin theory (with complex scalars being part of the spectrum) where this UV softening mechanism can be implemented also at the loop level. In particular, it is not clear if similar alternating series may appear in the bubble diagrams depending on cubic vertices with two higher spin fields.
} 


\section{Acknowledgments}

We thank X. Bekaert, S. Giombi and E. Joung for discussions and are also grateful to R. Metsaev, R. Roiban and E. Skvortsov for very useful comments on the draft. This work was supported by the ERC Advanced grant No. 290456. The work of AAT was also supported by the STFC Consolidated grant ST/L00044X/1 and the Russian Science Foundation grant 14-42-00047 associated with Lebedev Institute.

\section{A Relation between covariant and light-cone cubic vertices}

Our aim here will be to establish the relation between the covariant cubic vertex (3.3), (3.7) and the light-cone gauge one in [6]. We shall consider the case of $d=4$ Minkowski space with metric $\eta_{a b}=\operatorname{diag}(-,+,+,+)$ and define

$$
\begin{array}{lll}
x^{ \pm}=\frac{1}{\sqrt{2}}\left(x^{0} \pm x^{3}\right), & \mathrm{x}=\frac{1}{\sqrt{2}}\left(x^{1}+i x^{2}\right), & \overline{\mathrm{x}}=\frac{1}{\sqrt{2}}\left(x^{1}-i x^{2}\right), \\
\partial^{ \pm}=-\partial_{\mp}=\frac{1}{\sqrt{2}}\left(-\partial_{0} \pm \partial_{3}\right), & \partial=\frac{1}{\sqrt{2}}\left(\partial_{1}+i \partial_{2}\right), & \bar{\partial}=\frac{1}{\sqrt{2}}\left(\partial_{1}-i \partial_{2}\right),
\end{array}
$$

so that $d s^{2}=-2 d x^{+} d x^{-}+2 d \mathrm{x} d \overline{\mathrm{x}}$. Below we will label the $4 \mathrm{~d}$ indices by $a=0,1,2,3$ and by $(+,-, \mathrm{x}, \overline{\mathrm{x}})$ and also use $I, J, \ldots$ to label the $\mathrm{x}$ and $\overline{\mathrm{x}}$ directions. We will follow the standard notation

$$
\phi^{a(s)} \equiv \phi^{a_{1} a_{2} \ldots a_{s}}, \quad\left(\partial_{x}\right)_{a(s)} \equiv \frac{\partial}{\partial x^{a_{1}}} \frac{\partial}{\partial x^{a_{2}}} \cdots \frac{\partial}{\partial x^{a_{s}}},
$$

that is instead of writing all indices of the symmetric tensor, we just write one of them and indicate their number in brackets.

\section{A.1 Free fields in light-cone gauge}

The light-cone gauge for the Fronsdal fields that fixes the gauge freedom (2.8) completely corresponds to setting to zero all the components of the off-shell field $\phi^{a(s)}$ that have at least one upper "+" index

$$
\phi^{+-(n) I(s-n-1)}=0 .
$$

Here $n$ is the number of "-" indices and the remaining $s-n-1$ indices are the transverse ones $I=(\mathrm{x}, \overline{\mathrm{x}})$. In the light-cone gauge approach one usually assumes that derivatives $\partial^{+}$ of all fields and gauge parameters are non-vanishing, i.e. one can divide by $\partial^{+} .^{32}$

Let us review the consequences of this gauge condition when combined with partial set of the equations of motion (2.7) that allow one to express all of the components of the Fronsdal field in terms of just two independent ones. The components of e.o.m. (2.7) with two "+" indices give

$$
\partial^{+} \partial^{+} \phi_{m}^{m a(s-2)}=0 \Rightarrow \phi_{m}^{m a(s-2)}=0, \quad \text { i.e. } \quad \phi_{I}^{I a(s-2)}=0 .
$$

\footnotetext{
${ }^{32}$ Inverse powers of $\partial^{+}$need not be considered as indication of genuine non-localities but originate from solution of on-shell constraints.
} 
This implies that there are only two non-vanishing components of $\phi^{I(s)}$

$$
\varphi_{s} \equiv \phi^{\mathrm{x}(s)}, \quad \bar{\varphi}_{s} \equiv \phi^{\overline{\mathrm{x}}(s)} .
$$

The components of (2.7) with one "+" index give

$$
\partial^{+} \partial_{m} \phi^{m a(s-1)}=0 \quad \Rightarrow \quad \partial_{m} \phi^{m a(s-1)}=0,
$$

so the field is also transverse. Special cases of (A.6) give

$$
\phi^{-I(s-1)}=\frac{\partial_{J}}{\partial^{+}} \phi^{J I(s-1)}, \quad \phi^{--I(s-2)}=\frac{\partial_{J}}{\partial^{+}} \phi^{-J I(s-2)}, \quad \phi^{--I(s-2)}=\frac{\partial_{J} \partial_{J}}{\left(\partial^{+}\right)^{2}} \phi^{J J I(s-2)} .
$$

Proceeding in the same manner one gets

$$
\phi^{-(k) I(s-k)}=\frac{1}{\left(\partial^{+}\right)^{k}}\left(\partial_{J}\right)^{k} \phi^{J(k) I(s-k)} .
$$

Thus there are only two on-shell independent components of the $d=4$ Fronsdal field in the light-cone gauge - the two helicity fields (A.5). The equations of motion for them have the standard $\partial^{a} \partial_{a}=\square$ kinetic term, i.e. follow from the action ${ }^{33}$

$$
S_{2}\left[\varphi_{s}, \bar{\varphi}_{s}\right]=\int d^{4} x \bar{\varphi}_{s} \square \varphi_{s} .
$$

\section{A.2 Cubic interactions}

One imposes the same light-cone gauge (A.3) also at the interacting level. Interactions deform the free equations in a way that (A.4), (A.6), (A.8) now hold only up to terms linear in the coupling constant, e.g.,

$$
\phi_{m}^{m a(s-2)}=\mathcal{O}(g), \quad \partial_{m} \phi^{m a(s-1)}=\mathcal{O}(g) .
$$

These equations should be again used to express all of the auxiliary components of $\phi_{s}$ in terms of the two dynamical helicity fields $\varphi_{s}$ and $\bar{\varphi}_{s}$. The elimination of the auxiliary fields generates higher powers of $\varphi_{s}$ and $\bar{\varphi}_{s}$ even from the quadratic action but they contribute only to quartic and higher order interactions. Indeed, noting that except for the $\partial_{x}^{2}$-term all other terms in the free action (2.2) are at least bilinear in traces and divergences we conclude that

$$
\frac{s !}{2} \int d^{4} x\left[\phi_{s}\left(x, \partial_{u}\right) \hat{T} \hat{\mathcal{F}} \phi_{s}(x, u)\right]_{u=0}=\int d^{4} x \bar{\varphi}_{s} \square \varphi_{s}+\mathcal{O}\left(g^{2}\right) .
$$

Thus to find the cubic vertices for $\varphi_{s}$ field in the light-cone gauge from those in the covariant approach one just needs to plug (A.3)-(A.8) into the covariant cubic action.

\footnotetext{
${ }^{33}$ It is interesting to note the light-cone gauge action for free Fronsdal fields in $\mathrm{AdS}_{4}$ also has the same form as (A.9) [83], i.e. there are no extra "mass" terms present in the covariant expression (such terms appear though in $d>4)$.
} 
Let us start with the traceless-transverse part of the highest derivative cubic vertex in 4 dimensions $[13,14,16,36]^{34}$

$$
\begin{aligned}
S^{(3)}\left[\phi_{s_{1}}, \phi_{s_{2}}, \phi_{s_{3}}\right]=g_{s_{1} s_{2} s_{3}} \int d^{4} x[ & \left(\partial_{u_{1}} \cdot \partial_{x_{23}}\right)^{s_{1}}\left(\partial_{u_{2}} \cdot \partial_{x_{31}}\right)^{s_{2}}\left(\partial_{u_{3}} \cdot \partial_{x_{12}}\right)^{s_{3}} \\
& \left.\times \phi_{s_{1}}\left(x_{1}, u_{1}\right) \phi_{s_{2}}\left(x_{2}, u_{2}\right) \phi_{s_{3}}\left(x_{3}, u_{3}\right)\right]_{u_{i}=0} .
\end{aligned}
$$

The vertex in (3.3) is the special case of (A.12) corresponding to $s_{1}=0$. In the light-cone gauge one finds ${ }^{35}$

$$
\begin{aligned}
\left(\partial_{x_{12}}\right)_{a\left(s_{3}\right)} \phi^{a\left(s_{3}\right)}\left(x_{3}\right) & =\sum_{n=0}^{s} \frac{s !}{n !(s-n) !}\left(\partial_{x_{12}}\right)_{-(n)}\left(\partial_{x_{12}}\right)_{I\left(s_{3}-n\right)} \phi^{-(n) I\left(s_{3}-n\right)} \\
& =\sum_{n=0}^{s} \frac{(-1)^{n} s !}{n !(s-n) !}\left(\partial_{x_{12}}\right)^{+(n)}\left(\partial_{x_{12}}\right)_{I\left(s_{3}-n\right)} \frac{\partial_{3 J(n)}}{\left(\partial_{3}^{+}\right)^{n}} \phi^{J(n) I\left(s_{3}-n\right)} \\
& =\sum_{n=0}^{s} \frac{(-1)^{n} s !}{n !(s-n) !}\left(\partial_{x_{12}}^{+}\right)^{n} \bar{\partial}_{12}^{s_{3}-n} \frac{\bar{\partial}_{3}^{n}}{\left(\partial_{3}^{+}\right)^{n}} \varphi_{s_{3}}+\sum_{n=0}^{s} \frac{(-1)^{n} s !}{n !(s-n) !}\left(\partial_{x_{12}}^{+}\right)^{n} \partial_{12}^{s_{3}-n} \frac{\partial_{3}^{n}}{\left(\partial_{3}^{+}\right)^{n}} \bar{\varphi}_{s_{3}} \\
& =\left(\bar{\partial}_{12}-\frac{\partial_{12}^{+} \bar{\partial}_{3}}{\partial_{3}^{+}}\right)^{s_{3}} \varphi_{s_{3}}+\left(\partial_{12}-\frac{\partial_{12}^{+} \partial_{3}}{\partial_{3}^{+}}\right)^{s_{3}} \bar{\varphi}_{s_{3}} \\
& =\left(2 \frac{\bar{\partial}_{2} \partial_{1}^{+}-\bar{\partial}_{1} \partial_{2}^{+}}{\partial_{3}^{+}}\right)^{s_{3}} \varphi_{s_{3}}+\left(2 \frac{\partial_{2} \partial_{1}^{+}-\partial_{1} \partial_{2}^{+}}{\partial_{3}^{+}}\right)^{s_{3}} \bar{\varphi}_{s_{3}} .
\end{aligned}
$$

Following [6] let us introduce the notation

$$
\mathbb{P} \equiv \frac{1}{3}\left[\partial_{1}\left(\beta_{2}-\beta_{3}\right)+\partial_{2}\left(\beta_{3}-\beta_{1}\right)+\partial_{3}\left(\beta_{1}-\beta_{2}\right)\right], \quad \beta \equiv \partial_{-} .
$$

It is easy to see that

$$
\frac{\bar{\partial}_{2} \partial_{1}^{+}-\bar{\partial}_{1} \partial_{2}^{+}}{\partial_{3}^{+}}=-\frac{\overline{\mathbb{P}}}{\beta_{3}},
$$

so that (A.13) can be rewritten as

$$
\left(\partial_{x_{12}}\right)_{a\left(s_{3}\right)} \phi^{a\left(s_{3}\right)}\left(x_{3}\right)=\left(-2 \frac{\overline{\mathbb{P}}}{\beta_{3}}\right)^{s_{3}} \varphi_{s_{3}}+\left(-2 \frac{\mathbb{P}}{\beta_{3}}\right)^{s_{3}} \bar{\varphi}_{s_{3}} .
$$

As a result, we find that the covariant cubic vertex (A.12) written in the light-cone gauge becomes

$$
S_{3}=g_{s_{1} s_{2} s_{3}} \int d^{4} x\left(-2 \frac{\overline{\mathbb{P}}}{\beta_{1}}\right)^{s_{1}}\left(-2 \frac{\overline{\mathbb{P}}}{\beta_{2}}\right)^{s_{2}}\left(-2 \frac{\overline{\mathbb{P}}}{\beta_{3}}\right)^{s_{3}} \varphi_{s_{1}} \varphi_{s_{2}} \varphi_{s_{3}}+\ldots,
$$

where dots stand for analogous terms involving $\bar{\varphi}$. The light-cone gauge vertex found in [6] has exactly this form with ${ }^{36}$

$$
g_{s_{1} s_{2} s_{3}}=g \frac{\ell^{s_{1}+s_{2}+s_{3}-1}}{\left(s_{1}+s_{2}+s_{3}-1\right) !},
$$

where $g$ is an overall coupling constant and $\ell$ is an arbitrary dimension-length parameter.

\footnotetext{
${ }^{34}$ This vertex contains the maximal number of derivatives consistent with the condition of being non-zero when the fields are restricted to solutions of free equations of motion.

${ }^{35}$ Here in the last line we assume the possibility of integration by parts, i.e. $\partial_{3} \rightarrow-\partial_{1}-\partial_{2}$.

${ }^{36}$ The result (A.18) was derived in [6] for the special case when all spins entering the vertex are even and the more general case (with symmetric/antisymmetric internal indices on even/odd spin fields $\phi_{s}$ ) was considered in [7]. We shall assume that (A.17) holds for all values of the spins.
} 
Let us note that the vertex (A.12) has the following symmetry under the exchange of any two arguments

$$
S^{(3)}\left[\phi_{s_{1}}, \phi_{s_{2}}, \phi_{s_{3}}\right]=(-1)^{s_{1}+s_{2}+s_{3}} S^{(3)}\left[\phi_{s_{2}}, \phi_{s_{1}}, \phi_{s_{3}}\right] .
$$

To get a complete cubic interaction, this vertex has to be summed over all fields and this implicitly symmetrizes it over its arguments. This implies that the cubic vertex (A.12) contributes under summation over all spins only if the total spin of the fields is even, i.e. we may assume that the vertex with the total spin being odd may be effectively set to zero

$$
S^{(3)}\left[\phi_{s_{1}}, \phi_{s_{2}}, \phi_{s_{3}}\right]=0 \quad \text { if } \quad s_{1}+s_{2}+s_{3}=\text { odd } .
$$

\section{B Test of BCFW constructibility condition}

In this appendix we shall discuss if the above higher spin cubic vertices can be used to derive the quartic ones within the BCFW framework, i.e. if the BCFW constructibility condition is satisfied (cf. [21, 22]).

Let us consider a formal shift of two momenta

$$
p_{i} \rightarrow p_{i}(w)=p_{i}+w q, \quad p_{j} \rightarrow p_{j}(w)=p_{j}-w q
$$

where $w$ is a complex number and the vector $q$ satisfies

$$
q^{2}=0, \quad q \cdot p_{i}=0, \quad q \cdot p_{j}=0 .
$$

The BCFW constructibility condition [21] is that under such shift the total 4-point amplitude should vanish in the limit $w \rightarrow \infty$,

$$
\lim _{w \rightarrow \infty} \hat{\mathcal{A}}(w)=0 .
$$

Then assuming $\hat{\mathcal{A}}(w)$ is analytic it may have only poles at finite values of $w$. These poles should correspond to the values of $w$ for which the internal propagators that involve shifted momenta go on-shell. The residues at these poles are given by products of on-shell amplitudes resulting from the original one after cutting the propagator that goes on-shell under the shift. Using analyticity of the shifted amplitude $\mathcal{A}(w)$ as a function of $w$, one can then recursively express it in terms of the products of on-shell three-point amplitudes.

Let us apply these considerations to the tree-level four-scalar amplitude discussed in section 5. Under the shift (B.1) applied to $p_{1}$ and $p_{2}$ in (5.1) we find for the Mandelstam variables in $(5.2)$

$$
\mathrm{s}(w)=\mathrm{s}, \quad \mathrm{t}(w)=\mathrm{t}+w \Delta, \quad \mathrm{u}(w)=\mathrm{u}-w \Delta, \quad \Delta \equiv-2 q \cdot p_{1}^{\prime} .
$$

Then the constructibility condition (B.3) implies that the total 4-scalar amplitude containing the exchange and "contact" (4-vertex) contributions

$$
\hat{\mathcal{A}}(\mathrm{s}, \mathrm{t}, \mathrm{u})=\hat{\mathcal{A}}_{\mathrm{exch}}(\mathrm{s}, \mathrm{t}, \mathrm{u})+\mathcal{A}_{\mathrm{cont}}(\mathrm{s}, \mathrm{t}, \mathrm{u})
$$


should vanish at $\mathrm{t} \rightarrow \infty$. The total exchange part (5.11) of the 4-point amplitude corresponding to the s-channel expression in (5.9), (5.8) is

$$
\begin{aligned}
\hat{\mathcal{A}}_{\text {exch }}(\mathrm{s}, \mathrm{t}, \mathrm{u})= & -\frac{i}{\mathrm{~s}}\left[F\left(\frac{1}{2}(\sqrt{-\mathrm{u}}+\sqrt{\mathrm{t}})^{2}\right)+F\left(\frac{1}{2}(\sqrt{-\mathrm{u}}-\sqrt{\mathrm{t}})^{2}\right)\right] \\
& -\frac{i}{\mathrm{t}}\left[F\left(\frac{1}{2}(\sqrt{-\mathrm{u}}+\sqrt{\mathrm{s}})^{2}\right)+F\left(\frac{1}{2}(\sqrt{-\mathrm{u}}-\sqrt{\mathrm{s}})^{2}\right)\right] \\
& -\frac{i}{\mathrm{u}}\left[F\left(\frac{1}{2}(\sqrt{-\mathrm{s}}+\sqrt{\mathrm{t}})^{2}\right)+F\left(\frac{1}{2}(\sqrt{-\mathrm{s}}-\sqrt{\mathrm{t}})^{2}\right)\right] .
\end{aligned}
$$

To satisfy the constructibility condition, the contact contribution should cancel the exponential singularity (5.13) of this exchange amplitude (B.6) at $\mathrm{t} \rightarrow \infty$. Given that $F(z)$ in (5.8) is an entire function, then (under a natural assumption that "contact" contribution should not introduce new poles) the only remaining singularities of (B.6) will be poles at $\mathrm{t}=0$ and $\mathrm{t}=-\mathrm{s}$. Keeping only the contributions from these finite poles (as required by the assumption of BCFW constructibility), the total amplitude would then be

$$
\hat{\mathcal{A}}^{\prime}(\mathrm{s}, \mathrm{t}, \mathrm{u})=-i \frac{\mathrm{s}}{\mathrm{t}(\mathrm{s}+\mathrm{t})} F(2 \mathrm{~s}),
$$

where we used that $F(z)=F(-z)$ (see (5.8)). At the same time, applying the BCFW shift (B.1) to the momenta $p_{1}$ and $p_{1}^{\prime}$ in (5.1) and assuming a similar large $w$ singularity cancellation due to the contact term contribution we get instead

$$
\hat{\mathcal{A}}^{\prime \prime}(\mathrm{s}, \mathrm{t}, \mathrm{u})=-i \frac{\mathrm{t}}{\mathrm{s}(\mathrm{s}+\mathrm{t})} F(2 \mathrm{t}) \text {. }
$$

The two expressions (B.7) and (B.8) could agree provided $F(z)=\frac{c}{z^{2}}$ but this contradicts the actual form of $F$ in (5.8).

We are thus led to the conclusion that the cubic vertices (3.3) with the couplings (3.7) which led to the exchange amplitude (5.9) are inconsistent with the condition of BCFW constructibility.

\section{Details of summation over spins}

Here we shall evaluate some sums over spins appearing in section 6 . The bubble diagram contribution to the scalar self-energy (6.8) can be rewritten as

$$
\Sigma\left(k^{2}\right)=4 g^{2} \ell^{-2} \int \frac{d^{4} p}{p^{2}(p-k)^{2}}\left[2 \mathcal{S}_{1}+\frac{\left(2 k p-p^{2}\right)\left(p^{2}-k^{2}\right)}{(2 k-p)^{2}(k+p)^{2}} \mathcal{S}_{2}\right]
$$

where

$$
\mathcal{S}_{1} \equiv \sum_{\substack{m, n=0 \\ m+n=\text { even }}}^{\infty} \frac{x^{m} y^{n}}{((m+n-1) !)^{2}}, \quad \mathcal{S}_{2} \equiv \sum_{\substack{m, n=0 \\ m+n=\text { even }}}^{\infty} \frac{m^{2} n^{2} x^{m} y^{n}}{((m+n-1) !)^{2}},
$$

and $x \equiv \frac{1}{2} \ell^{2}(2 k-p)^{2}, y \equiv \frac{1}{2} \ell^{2}(k+p)^{2}$ as in (6.11). To evaluate $\mathcal{S}_{1}$ let us first introduce the new variables

$$
u \equiv x y, \quad v=\frac{x}{y}
$$


and then rearrange the series to sum over $m$ and $n$ obeying $m+n=2 r$ with fixed integer $r$ first

$\mathcal{S}_{1}=\sum_{\substack{m, n=0 \\ m+n=2 r}}^{\infty} \frac{u^{(m+n) / 2} v^{(m-n) / 2}}{((m+n-1) !)^{2}}=\sum_{r=0}^{\infty} \frac{u^{r} v^{r}}{((2 r-1) !)^{2}} \sum_{n=0}^{2 r} v^{-n}=\sum_{r=0}^{\infty} \frac{u^{r} v^{r}}{((2 r-1) !)^{2}} \frac{1-v^{-(2 r+1)}}{1-v^{-1}}$.

After some simple manipulations we find

$$
\begin{aligned}
\mathcal{S}_{1} & =\frac{v}{v-1} \sum_{r=0}^{\infty} \frac{x^{2 r}}{((2 r-1) !)^{2}}-\frac{1}{v-1} \sum_{r=0}^{\infty} \frac{y^{2 r}}{((2 r-1) !)^{2}} \\
& =\frac{x^{2}}{2(x-y)}\left[I_{0}(2 \sqrt{x})-J_{0}(2 \sqrt{x})\right]-\frac{y^{2}}{2(x-y)}\left[I_{0}(2 \sqrt{y})-J_{0}(2 \sqrt{y})\right],
\end{aligned}
$$

where $J_{0}(z)$ and $I_{0}(z)$ are the Bessel functions in (5.7).

Similarly, for the second sum we find

$$
\mathcal{S}_{2}=\sum_{\substack{m, n=0 \\ m+n=2 r}}^{\infty} \frac{m^{2} n^{2}}{((m+n-1) !)^{2}} u^{(m+n) / 2} v^{(m-n) / 2}=\sum_{r=0}^{\infty} \frac{u^{r} v^{r}}{((2 r-1) !)^{2}} \sum_{n=0}^{2 r} n^{2}(2 r-n)^{2} v^{-n}
$$

Let us set $j \equiv 2 r=m+n$. Then the sum over $n$ acquires the following form

$$
\begin{aligned}
\sum_{n=0}^{j} n^{2}(j-n)^{2} v^{-n} & =\frac{v}{(v-1)^{5}}\left[A(v, j)-v^{-j} B(v, j)\right] \\
A(v, j) & =j^{2} v^{3}+2 j v^{3}+v^{3}-j^{2} v^{2}+6 j v^{2}+11 v^{2}-j^{2} v-6 j v+11 v+j^{2}-2 j+1 \\
B(v, j) & =j^{2} v^{3}-2 j v^{3}+v^{3}-j^{2} v^{2}-6 j v^{2}+11 v^{2}-j^{2} v+6 j v+11 v+j^{2}-2 j+1 .
\end{aligned}
$$

It is convenient to represent $A$ and $B$ as

$$
\begin{aligned}
& A(v, j)=(j-1)(j-2)(v-1)^{2}(v+1)+(j-1)(v-1)\left(v^{2}-8 v-5\right)+4\left(v^{2}+4 v+1\right), \\
& B(v, j)=(j-1)(j-2)(v-1)^{2}(v+1)+(j-1)(v-1)\left(5 v^{2}+8 v-1\right)+4 v\left(v^{2}+4 v+1\right) .
\end{aligned}
$$

Then $\mathcal{S}_{2}$ may be written as

$$
\mathcal{S}_{2}=\frac{v}{(v-1)^{5}} \sum_{r=0}^{\infty} \frac{x^{2 r}}{((2 r-1) !)^{2}} A(v, 2 r)-\frac{v}{(v-1)^{5}} \sum_{r=0}^{\infty} \frac{y^{2 r}}{((2 r-1) !)^{2}} B(v, 2 r) .
$$

Substituting here the expressions for $A$ and $B$ we obtain

$$
\begin{aligned}
\mathcal{S}_{2}=\frac{v}{(v-1)^{5}}[ & (v-1)^{2}(v+1) \sum_{r=0}^{\infty} \frac{x^{2 r}}{(2 r-1) !(2 r-3) !}-4 v\left(v^{2}+4 v+1\right) \sum_{r=0}^{\infty} \frac{y^{2 r}}{((2 r-1) !)^{2}} \\
& +(v-1)\left(v^{2}-8 v-5\right) \sum_{r=0}^{\infty} \frac{x^{2 r}}{(2 r-1) !(2 r-2) !}+4\left(v^{2}+4 v+1\right) \sum_{r=0}^{\infty} \frac{x^{2 r}}{((2 r-1) !)^{2}} \\
& \left.-(v-1)^{2}(v+1) \sum_{r=0}^{\infty} \frac{y^{2 r}}{(2 r-1) !(2 r-3) !}-(v-1)\left(5 v^{2}+8 v-1\right) \sum_{r=0}^{\infty} \frac{x^{2 r}}{(2 r-1) !(2 r-2) !}\right] .
\end{aligned}
$$


Using (5.7) to perform the summations and expressing the result back in terms of $x$ and $y$ in (C.3) we find

$$
\begin{aligned}
\mathcal{S}_{2}= & \frac{x^{3} y(x+y)}{2(x-y)^{3}}\left[I_{2}(2 \sqrt{x})-J_{2}(2 \sqrt{x})\right]+\frac{x^{2} y\left(x^{2}-8 x y-5 y^{2}\right) \sqrt{x}}{2(x-y)^{4}}\left[I_{1}(2 \sqrt{x})+J_{1}(2 \sqrt{x})\right] \\
& +\frac{2 x^{2} y^{2}\left(x^{2}+4 x y+y^{2}\right)}{(x-y)^{5}}\left[I_{0}(2 \sqrt{x})-J_{0}(2 \sqrt{x})\right]+(x \leftrightarrow y) .
\end{aligned}
$$

As a result, we get the following expression for (C.1) which is equivalent to (6.9), (6.10)

$$
\begin{aligned}
\Sigma\left(k^{2}\right)=4 g^{2} \ell^{-2} \int \frac{d^{4} p}{p^{2}(p-k)^{2}}( & {\left[\frac{x^{2}}{x-y}\left[I_{0}(2 \sqrt{x})-J_{0}(2 \sqrt{x})\right]+(x \leftrightarrow y)\right] } \\
& +\frac{\left(2 k p-p^{2}\right)\left(p^{2}-k^{2}\right)}{(2 k-p)^{2}(k+p)^{2}}\left[\frac{x^{3} y(x+y)}{2(x-y)^{3}}\left[I_{2}(2 \sqrt{x})-J_{2}(2 \sqrt{x})\right]\right. \\
& +\frac{x^{2} y\left(x^{2}-8 x y-5 y^{2}\right) \sqrt{x}}{2(x-y)^{4}}\left[I_{1}(2 \sqrt{x})+J_{1}(2 \sqrt{x})\right] \quad(\text { C. } 8) \\
& \left.\left.+\frac{2 x^{2} y^{2}\left(x^{2}+4 x y+y^{2}\right)}{(x-y)^{5}}\left[I_{0}(2 \sqrt{x})-J_{0}(2 \sqrt{x})\right]+(x \leftrightarrow y)\right]\right) .
\end{aligned}
$$

Let us note that (C.8) is not directly applicable for $x=y$ because intermediate summations over powers of $v$ resulted in a spurious pole at $v=1$. The $x=y$ limit is actually regular as one can show by expanding the numerators in powers of $(x-y)$ and checking that all factors of $(x-y)$ in the denominator get cancelled. Equivalently, one may just evaluate the above sums explicitly for $v=1$. This gives

$$
\begin{aligned}
\left.\mathcal{S}_{1}\right|_{x=y}= & \frac{1}{2}(\sqrt{x})^{3}\left[I_{1}(2 \sqrt{x})+J_{1}(2 \sqrt{x})\right]+x\left[I_{0}(2 \sqrt{x})-J_{0}(2 \sqrt{x})\right] \\
\left.\mathcal{S}_{2}\right|_{x=y}= & \frac{1}{60}(\sqrt{x})^{7}\left[I_{5}(2 \sqrt{x})+J_{5}(2 \sqrt{x})\right]+\frac{1}{4} x^{3}\left[I_{4}(2 \sqrt{x})-J_{4}(2 \sqrt{x})\right] \\
& +\frac{13}{12}(\sqrt{x})^{5}\left[I_{3}(2 \sqrt{x})+J_{3}(2 \sqrt{x})\right]+\frac{3}{2} x^{2}\left[I_{2}(2 \sqrt{x})-J_{2}(2 \sqrt{x})\right] \\
& +\frac{1}{2}(\sqrt{x})^{3}\left[I_{1}(2 \sqrt{x})+J_{1}(2 \sqrt{x})\right] .
\end{aligned}
$$

As a result, in the $x=y$ limit (which corresponds according to (6.11) to the $k^{2}=0$ value of the self-energy correction and thus determines the leading UV $p \rightarrow \infty$ behaviour of the integrand in (C.1)) one finds the expression given in (6.12).

Open Access. This article is distributed under the terms of the Creative Commons Attribution License (CC-BY 4.0), which permits any use, distribution and reproduction in any medium, provided the original author(s) and source are credited.

\section{References}

[1] X. Bekaert, N. Boulanger and P. Sundell, How higher-spin gravity surpasses the spin two barrier: no-go theorems versus yes-go examples, Rev. Mod. Phys. 84 (2012) 987 [arXiv: 1007.0435] [INSPIRE]. 
[2] A.K.H. Bengtsson, I. Bengtsson and L. Brink, Cubic interaction terms for arbitrary spin, Nucl. Phys. B 227 (1983) 31 [inSPIRE].

[3] F.A. Berends, G.J.H. Burgers and H. Van Dam, On spin three selfinteractions, Z. Phys. C 24 (1984) 247 [INSPIRE].

[4] F.A. Berends, G.J.H. Burgers and H. Van Dam, On the theoretical problems in constructing interactions involving higher spin massless particles, Nucl. Phys. B 260 (1985) 295 [INSPIRE].

[5] A.K.H. Bengtsson, I. Bengtsson and N. Linden, Interacting higher spin gauge fields on the light front, Class. Quant. Grav. 4 (1987) 1333 [INSPIRE].

[6] R.R. Metsaev, Poincaré invariant dynamics of massless higher spins: fourth order analysis on mass shell, Mod. Phys. Lett. A 6 (1991) 359 [INSPIRE].

[7] R.R. Metsaev, S-matrix approach to massless higher spins theory II: the case of internal symmetry, Mod. Phys. Lett. A 6 (1991) 2411 [INSPIRE].

[8] R.R. Metsaev, Generating function for cubic interaction vertices of higher spin fields in any dimension, Mod. Phys. Lett. A 8 (1993) 2413 [INSPIRE].

[9] R.R. Metsaev, Cubic interaction vertices of massive and massless higher spin fields, Nucl. Phys. B 759 (2006) 147 [hep-th/0512342] [INSPIRE].

[10] A. Fotopoulos and M. Tsulaia, Gauge invariant Lagrangians for free and interacting higher spin fields: a review of the BRST formulation, Int. J. Mod. Phys. A 24 (2009) 1 [arXiv:0805.1346] [INSPIRE].

[11] Y.M. Zinoviev, On spin 3 interacting with gravity, Class. Quant. Grav. 26 (2009) 035022 [arXiv:0805.2226] [INSPIRE].

[12] N. Boulanger, S. Leclercq and P. Sundell, On the uniqueness of minimal coupling in higher-spin gauge theory, JHEP 08 (2008) 056 [arXiv:0805.2764] [INSPIRE].

[13] R. Manvelyan, K. Mkrtchyan and W. Rühl, General trilinear interaction for arbitrary even higher spin gauge fields, Nucl. Phys. B 836 (2010) 204 [arXiv: 1003.2877] [INSPIRE].

[14] A. Sagnotti and M. Taronna, String lessons for higher-spin interactions, Nucl. Phys. B 842 (2011) 299 [arXiv: 1006.5242] [INSPIRE].

[15] A. Fotopoulos and M. Tsulaia, On the tensionless limit of string theory, off-shell higher spin interaction vertices and BCFW recursion relations, JHEP 11 (2010) 086 [arXiv:1009.0727] [INSPIRE].

[16] R. Manvelyan, K. Mkrtchyan and W. Ruehl, A generating function for the cubic interactions of higher spin fields, Phys. Lett. B 696 (2011) 410 [arXiv: 1009.1054] [InSPIRE].

[17] M. Taronna, Higher-spin interactions: four-point functions and beyond, JHEP 04 (2012) 029 [arXiv: 1107.5843] [INSPIRE].

[18] A. Sagnotti, Notes on strings and higher spins, J. Phys. A 46 (2013) 214006 [arXiv: 1112.4285] [INSPIRE].

[19] S. Weinberg, The quantum theory of fields. Vol. 1: Foundations, Cambridge University Press, Cambridge U.K. (2005) [INSPIRE].

[20] R. Britto, F. Cachazo, B. Feng and E. Witten, Direct proof of tree-level recursion relation in Yang-Mills theory, Phys. Rev. Lett. 94 (2005) 181602 [hep-th/0501052] [INSPIRE]. 
[21] P. Benincasa and F. Cachazo, Consistency conditions on the S-matrix of massless particles, arXiv:0705.4305 [INSPIRE].

[22] P. Benincasa and E. Conde, Exploring the S-matrix of massless particles, Phys. Rev. D 86 (2012) 025007 [arXiv: 1108.3078] [InSPIRE].

[23] D.A. McGady and L. Rodina, Higher-spin massless S-matrices in four-dimensions, Phys. Rev. D 90 (2014) 084048 [arXiv:1311.2938] [InSPIRE].

[24] M. Beccaria and A.A. Tseytlin, On higher spin partition functions, J. Phys. A 48 (2015) 275401 [arXiv:1503.08143] [inSPIRE].

[25] A. Campoleoni, H.A. Gonzalez, B. Oblak and M. Riegler, Rotating higher spin partition functions and extended BMS symmetries, JHEP 04 (2016) 034 [arXiv:1512.03353] [INSPIRE].

[26] A.A. Tseytlin, On partition function and Weyl anomaly of conformal higher spin fields, Nucl. Phys. B 877 (2013) 598 [arXiv: 1309.0785] [inSPIRE].

[27] E. Joung, S. Nakach and A.A. Tseytlin, Scalar scattering via conformal higher spin exchange, JHEP 02 (2016) 125 [arXiv:1512.08896] [INSPIRE].

[28] S.J. Parke, Absence of particle production and factorization of the S-matrix in (1+1)-dimensional models, Nucl. Phys. B 174 (1980) 166 [INSPIRE].

[29] E.S. Fradkin and M.A. Vasiliev, Cubic interaction in extended theories of massless higher spin fields, Nucl. Phys. B 291 (1987) 141 [INSPIRE].

[30] E.S. Fradkin and M.A. Vasiliev, On the gravitational interaction of massless higher spin fields, Phys. Lett. B 189 (1987) 89 [INSPIRE].

[31] M.A. Vasiliev, Consistent equation for interacting gauge fields of all spins in (3+1)-dimensions, Phys. Lett. B 243 (1990) 378 [INSPIRE].

[32] M.A. Vasiliev, Nonlinear equations for symmetric massless higher spin fields in $(A) d S_{d}$, Phys. Lett. B 567 (2003) 139 [hep-th/0304049] [INSPIRE].

[33] S. Giombi and X. Yin, Higher spin gauge theory and holography: the three-point functions, JHEP 09 (2010) 115 [arXiv:0912.3462] [INSPIRE].

[34] S. Giombi and X. Yin, The higher spin/vector model duality, J. Phys. A 46 (2013) 214003 [arXiv: 1208.4036] [INSPIRE].

[35] N. Boulanger, P. Kessel, E.D. Skvortsov and M. Taronna, Higher spin interactions in four-dimensions: Vasiliev versus Fronsdal, J. Phys. A 49 (2016) 095402 [arXiv: 1508.04139] [INSPIRE].

[36] E. Joung and M. Taronna, Cubic interactions of massless higher spins in (A)dS: metric-like approach, Nucl. Phys. B 861 (2012) 145 [arXiv:1110.5918] [INSPIRE].

[37] S. Giombi, I.R. Klebanov, S.S. Pufu, B.R. Safdi and G. Tarnopolsky, AdS description of induced higher-spin gauge theory, JHEP 10 (2013) 016 [arXiv:1306.5242] [INSPIRE].

[38] S. Giombi and I.R. Klebanov, One loop tests of higher spin AdS/CFT, JHEP 12 (2013) 068 [arXiv: 1308.2337] [INSPIRE].

[39] S. Giombi, I.R. Klebanov and B.R. Safdi, Higher spin $A d S_{d+1} / C F T_{d}$ at one loop, Phys. Rev. D 89 (2014) 084004 [arXiv:1401.0825] [INSPIRE]. 
[40] S. Giombi, I.R. Klebanov and A.A. Tseytlin, Partition functions and Casimir energies in higher spin $A d S_{d+1} / C F T_{d}$, Phys. Rev. D 90 (2014) 024048 [arXiv: 1402.5396] [INSPIRE].

[41] M. Beccaria, X. Bekaert and A.A. Tseytlin, Partition function of free conformal higher spin theory, JHEP 08 (2014) 113 [arXiv: 1406.3542] [INSPIRE].

[42] M. Beccaria and A.A. Tseytlin, Higher spins in $A d S_{5}$ at one loop: vacuum energy, boundary conformal anomalies and AdS/CFT, JHEP 11 (2014) 114 [arXiv:1410.3273] [INSPIRE].

[43] I.R. Klebanov and A.M. Polyakov, AdS dual of the critical $O(N)$ vector model, Phys. Lett. B 550 (2002) 213 [hep-th/0210114] [INSPIRE].

[44] R.G. Leigh and A.C. Petkou, Holography of the $\mathcal{N}=1$ higher spin theory on AdS , JHEP 06 (2003) 011 [hep-th/0304217] [INSPIRE].

[45] E. Sezgin and P. Sundell, Holography in $4 D$ (super) higher spin theories and a test via cubic scalar couplings, JHEP 07 (2005) 044 [hep-th/0305040] [INSPIRE].

[46] S. Giombi and X. Yin, Higher spins in AdS and twistorial holography, JHEP 04 (2011) 086 [arXiv: 1004.3736] [INSPIRE].

[47] J. Maldacena and A. Zhiboedov, Constraining conformal field theories with a higher spin symmetry, J. Phys. A 46 (2013) 214011 [arXiv:1112.1016] [INSPIRE].

[48] N. Colombo and P. Sundell, Higher spin gravity amplitudes from zero-form charges, arXiv: 1208.3880 [INSPIRE].

[49] V.E. Didenko and E.D. Skvortsov, Exact higher-spin symmetry in CFT: all correlators in unbroken Vasiliev theory, JHEP 04 (2013) 158 [arXiv:1210.7963] [INSPIRE].

[50] O.A. Gelfond and M.A. Vasiliev, Operator algebra of free conformal currents via twistors, Nucl. Phys. B 876 (2013) 871 [arXiv:1301.3123] [INSPIRE].

[51] X. Bekaert, J. Erdmenger, D. Ponomarev and C. Sleight, Towards holographic higher-spin interactions: four-point functions and higher-spin exchange, JHEP 03 (2015) 170 [arXiv: 1412.0016] [INSPIRE].

[52] X. Bekaert, J. Erdmenger, D. Ponomarev and C. Sleight, Quartic AdS interactions in higher-spin gravity from conformal field theory, JHEP 11 (2015) 149 [arXiv:1508.04292] [INSPIRE].

[53] C. Sleight and M. Taronna, Higher spin interactions from conformal field theory: the complete cubic couplings, Phys. Rev. Lett. 116 (2016) 181602 [arXiv:1603.00022] [INSPIRE].

[54] A.C. Petkou, Evaluating the AdS dual of the critical $O(N)$ vector model, JHEP 03 (2003) 049 [hep-th/0302063] [INSPIRE].

[55] M. Porrati, Higgs phenomenon for $4 D$ gravity in anti-de Sitter space, JHEP 04 (2002) 058 [hep-th/0112166] [INSPIRE].

[56] M.J. Duff, J.T. Liu and H. Sati, Complementarity of the Maldacena and Karch-Randall pictures, Phys. Rev. D 69 (2004) 085012 [hep-th/0207003] [InSPIRE].

[57] R. Manvelyan, K. Mkrtchyan and W. Rühl, Ultraviolet behaviour of higher spin gauge field propagators and one loop mass renormalization, Nucl. Phys. B 803 (2008) 405 [arXiv:0804.1211] [INSPIRE].

[58] J. Penedones, Writing CFT correlation functions as AdS scattering amplitudes, JHEP 03 (2011) 025 [arXiv: 1011.1485] [INSPIRE]. 
[59] E.D. Skvortsov, On (un)broken higher-spin symmetry in vector models, arXiv:1512.05994 [INSPIRE].

[60] S. Giombi and V. Kirilin, Anomalous dimensions in CFT with weakly broken higher spin symmetry, arXiv:1601.01310 [INSPIRE].

[61] Y. Hikida, The masses of higher spin fields on AdS $S_{4}$ and conformal perturbation theory, arXiv:1601.01784 [INSPIRE].

[62] V. Bashmakov, M. Bertolini, L. Di Pietro and H. Raj, Scalar multiplet recombination at large- $N$ and holography, arXiv:1603.00387 [INSPIRE].

[63] M.A. Vasiliev, Cubic vertices for symmetric higher-spin gauge fields in $(A) d S_{d}$, Nucl. Phys. B 862 (2012) 341 [arXiv:1108.5921] [INSPIRE].

[64] N. Boulanger, D. Ponomarev and E.D. Skvortsov, Non-Abelian cubic vertices for higher-spin fields in $A d S_{d}$, JHEP 05 (2013) 008 [arXiv: 1211.6979] [INSPIRE].

[65] E.S. Fradkin and M.A. Vasiliev, Candidate to the role of higher-spin symmetry, Annals Phys. 177 (1987) 63 [INSPIRE].

[66] N. Boulanger, D. Ponomarev, E.D. Skvortsov and M. Taronna, On the uniqueness of higher-spin symmetries in AdS and CFT, Int. J. Mod. Phys. A 28 (2013) 1350162 [arXiv: 1305.5180] [INSPIRE].

[67] M.A. Vasiliev, Dynamics of massless higher spins in the second order in curvatures, Phys. Lett. B 238 (1990) 305 [inSPIRE].

[68] D. Polyakov, Higher spins and open strings: quartic interactions, Phys. Rev. D 83 (2011) 046005 [arXiv:1011.0353] [INSPIRE].

[69] P. Dempster and M. Tsulaia, On the structure of quartic vertices for massless higher spin fields on Minkowski background, Nucl. Phys. B 865 (2012) 353 [arXiv:1203.5597] [INSPIRE].

[70] I.L. Buchbinder and V.A. Krykhtin, Quartic interaction vertex in the massive integer higher spin field theory in a constant electromagnetic field, Eur. Phys. J. C 75 (2015) 454 [arXiv: 1507.03723] [INSPIRE].

[71] C. Fronsdal, Massless fields with integer spin, Phys. Rev. D 18 (1978) 3624 [InSPIRE].

[72] G. Barnich and M. Henneaux, Consistent couplings between fields with a gauge freedom and deformations of the master equation, Phys. Lett. B 311 (1993) 123 [hep-th/9304057] [INSPIRE].

[73] X. Bekaert, N. Boulanger and S. Cnockaert, Spin three gauge theory revisited, JHEP 01 (2006) 052 [hep-th/0508048] [INSPIRE].

[74] N. Boulanger and S. Leclercq, Consistent couplings between spin-2 and spin-3 massless fields, JHEP 11 (2006) 034 [hep-th/0609221] [INSPIRE].

[75] X. Bekaert, N. Boulanger and S. Leclercq, Strong obstruction of the Berends-Burgers-van Dam spin-3 vertex, J. Phys. A 43 (2010) 185401 [arXiv:1002.0289] [INSPIRE].

[76] E. Joung and M. Taronna, Cubic-interaction-induced deformations of higher-spin symmetries, JHEP 03 (2014) 103 [arXiv:1311.0242] [INSPIRE].

[77] B. de Wit and D.Z. Freedman, Systematics of higher spin gauge fields, Phys. Rev. D 21 (1980) 358 [inSPIRE]. 
[78] D. Francia, J. Mourad and A. Sagnotti, Current exchanges and unconstrained higher spins, Nucl. Phys. B 773 (2007) 203 [hep-th/0701163] [INSPIRE].

[79] X. Bekaert, E. Joung and J. Mourad, On higher spin interactions with matter, JHEP 05 (2009) 126 [arXiv:0903.3338] [INSPIRE].

[80] M.B. Green, J.H. Schwarz and E. Witten, Superstring theory. Vol. 1: Introduction, Cambridge University Press, Cambridge U.K. (1987) [InSPIRE].

[81] M. Taronna, Pseudo-local theories: a functional class proposal, in Proceedings of the International Workshop on Higher Spin Gauge Theories, Singapore, 4-6 Nov 2015 [arXiv: 1602.08566] [INSPIRE].

[82] X. Bekaert, J. Erdmenger, D. Ponomarev and C. Sleight, Bulk quartic vertices from boundary four-point correlators, in Proceedings of the International Workshop on Higher Spin Gauge Theories, Singapore, 4-6 Nov 2015 [arXiv: 1602.08570] [INSPIRE].

[83] R.R. Metsaev, Light cone form of field dynamics in anti-de Sitter space-time and AdS/CFT correspondence, Nucl. Phys. B 563 (1999) 295 [hep-th/9906217] [InSPIRE]. 